Research Article

\title{
Shannon-Cosine Wavelet Precise Integration Method for Locust Slice Image Mixed Denoising
}

\author{
Haihua Wang $\mathbb{D}^{1,2}$ Xinxin Zhang, ${ }^{1,2}$ and Shuli Mei $\mathbb{D}^{1,2}$ \\ ${ }^{1}$ China Agricultural University, East Campus, Postbox 53, 17 Qinghua Donglu Road, Haidian District, Beijing 100083, China \\ ${ }^{2}$ College of Information and Electrical Engineering, China Agricultural University, Beijing 100083, China
}

Correspondence should be addressed to Shuli Mei; meishuli@163.com

Received 23 December 2019; Revised 11 April 2020; Accepted 27 May 2020; Published 14 July 2020

Academic Editor: Bogdan Smolka

Copyright ( 92020 Haihua Wang et al. This is an open access article distributed under the Creative Commons Attribution License, which permits unrestricted use, distribution, and reproduction in any medium, provided the original work is properly cited.

A novel denoising method for removing mixed noise from locust slice images is proposed by means of Shannon-cosine wavelet and the nonlinear variational model for the image processing. This method includes two parts that are the sparse representation of the slice images and the novel numerical algorithm for solving the variation model on image denoising based on the sparse representation. In the first part, a parametric Shannon-cosine wavelet function is introduced to construct the multiscale wavelet transform matrix, which is applied to represent the slice images sparsely by adjusting the parameters adaptively based on the texture of the locust slice images. By multiplying the matrix with the signal, the multiscale wavelet transform coefficients of the signal can be obtained at one time, which can be used to identify the salt-and-pepper noises in the slice images. This ensures that the salt-and-pepper noise points are kept away from the sparse representation of the slice images. In the second part, a semianalytical method on solving the system of the nonlinear differential equations is constructed based on the sparse representation of the slice images, which is named the sparse wavelet precise integration method (SWPIM). Substituting the sparse representation of the slice images into the Perona-Malik model which is the famous edge-preserving smoothing model for removing the Gaussian noises of the biomedical images, a system of nonlinear differential equations is obtained, whose scale is far smaller than the one obtained by the difference method. The numerical experiments show that both the values of SSIM and PSNR of the denoised locust slice images are better than the classical methods besides the algorithm efficiency.

\section{Introduction}

The main purpose of locust microscopy experiments is to reveal the working mechanism of locust reproduction and pesticide extermination by studying the structure of locust cells and their neural tissues [1]. In addition, it is helpful for the research and development of biological pesticides and reduces the use of chemical pesticides [2]. Locust slice images are quite complex such as textures, edges, and structures, and slice tissue structures cannot be acquired repeatedly [3]. The locust slice images have the characteristics of biomedical image segmentation smoothing [4]. The salt-and-pepper noise and Gaussian noise can not only significantly deteriorate the quality of locust slice images but also bring some difficulty to the subsequent image analysis such as image segmentation, edge detection, and classification. How to effectively remove salt-and-pepper noise and Gaussian noise in locust slice images has been an important research topic in the field of image processing.

During the past several decades, many techniques have been developed to restore the corrupted image, but research on locust slice images is lacking. Most traditional filtering methods are designed for a certain type of noise, and the effect of removing salt-and-pepper and Gaussian mixed noises is not ideal. Among the traditional denoising methods, the standard median filter [5] is one of the most popular nonlinear filters for the removal of salt-and-pepper noise in terms of its good denoising capability and computational efficiency, but high noise density will lead to unsatisfactory denoising results with serious loss of image details. Wiener filtering [6] is a popular type of Gaussian noise removing technique with good denoising 
performance. However, it cannot remove the salt-andpepper noise well due to a larger mean square error with the original image. Mean filtering [7] is not very effective in removing salt-and-pepper noise, and its application range is limited. Gaussian filtering [8] will damage the edge information of the image when denoising, and the denoising performance is poor. During the denoising process, the wavelet transform [9] is prone to oscillation, and the edge and texture details cannot be well preserved. Although the above algorithm can achieve good results when removing the special noise, it is not suitable for mixed noise to meet some situations. The detection and nonlocal mean denoising method based on robust singularity rate proposed in [10] had a large time complexity in the block search stage. In [11], combined adaptive median filtering (AMF) with improved sparse representation to remove mixed noise is presented. It uses the AMF to remove salt-and-pepper noise and then removes Gaussian noise by means of improved K-singular value decomposition (K-SVD) dictionary learning method and backtracking-based adaptive orthogonal matching pursuit (BAOMP) sparse coding method. In [12], the new mixed noise removal scheme is based on a weighted averaging of pixels contained in a filtering block. The main advantage of the proposed scheme is that the new similarity measure is not influenced by the outliers injected into the image by the impulsive noise, and the averaging process ensures the effectiveness of the new filter in the reduction of Gaussian noise. The anisotropic dynamic diffusion model proposed in [13] could not only effectively remove noise but also retain more texture details. Generally speaking, the P-M model is a nonlinear PDE that can identify textures and edge regions. After the denoising process, the texture and edges can be effectively retained to achieve the effect of edge preservation.

Image denoising is an extensively studied problem in the image processing community and continues to attract researchers who aim to perform better restoration in the presence of noise. Both of the Gaussian and salt-and-pepper noises are the common types of noises. Locust slice images are easy to be corrupted by salt-and-pepper noise due to bit errors in the process of image acquisition and transmission. The locust slice images may also be corrupted by Gaussian noise because the transmittance of different particles to light is Gaussian distribution. In this paper, the problem of image denoising with mixed noise is considered, in which the image is contaminated by both Gaussian noise and salt-andpepper noise. However, the variation method cannot effectively remove salt-and-pepper noise, and all other existed filtering algorithms are all most with the limitations. So, a novel denoising method for removing mixed noise from locust slice images is proposed by means of Shannon-cosine wavelet and the nonlinear variational model for the image processing. This method includes two parts that are the sparse representation of the slice images and the novel numerical algorithm for solving the variation model on image denoising based on the sparse representation. In order to accurately recognize Gaussian noise and image contour of the image, a method is designed to calculate the adaptive selection parameter using image contrast. With this new image removing method, the mixed noise of locust slice images can be processed precisely and efficiency.

\section{Sparse Representation of the Slice Images}

In the recent decades, many wavelets which have compact support, smoothness, and other properties have been constructed. Cattani studied the properties of the Shannon wavelet function, which possesses many advantages such as orthogonality, continuity, and differentiability [14, 15]. A perceived disadvantage of the Shannon scaling function is that it tends to zero quite slowly as $|x| \longrightarrow \infty$ [16]. Shannon-Gabor wavelet constructed by Aldroubi et al. $[17,18]$ is a Shannon wavelet with Gauss window, which is being called quasiwavelet with limited use. Recently, the Shannon-cosine wavelet [19] was proposed with the characteristic of compact support, which inherits all the excellent characteristics of the Shannon wavelet and the normalized characteristics of the Shannon function. Furthermore, Shannon-cosine wavelet has been used to solve the fractional calculus problems in the recent years [20]. Therefore, Shannon-cosine wavelet is employed to construct the multiscale interpolator operator in our research, which can be expressed as follows:

$$
S_{C}(x)=\frac{\sin (\pi x)}{\pi x} T_{N}^{m}(x) R_{N}(x)
$$

where

$$
\begin{aligned}
& T_{N}^{m}(x)=\sum_{n=0}^{m}\left(a_{n} \cos \frac{2 n \pi x}{N}\right), \\
& R_{N}(x)= {\left[\chi\left(x+\frac{N}{2}\right)-\chi\left(x-\frac{N}{2}\right)\right], } \\
& \chi(x)= \begin{cases}0, & (x<0), \\
\frac{1}{2}, & (x=0), \\
1, & (x>0),\end{cases}
\end{aligned}
$$

where $R_{N}(x)$ is the matrix pulse function on $[-N / 2, N / 2]$ and $N$ is a constant associated with the support interval. $\chi(x)$ denotes the Heaviside function. The pulse function not only gives the Shannon-cosine scale function tight support but also has no longer the continuity of $x= \pm N / 2$ at the endpoints. At the same time, the normalized property at the endpoint can be satisfied by selecting the parameters $a_{i}(i=$ $0,1, \cdots, m)$ and $N$ [21]. So, the Shannon-cosine wavelet is more suitable to locust slice images with excellent properties such as small support interval, compact support, and normalization.

2.1. Construction Schemes of Multiscale Interpolation Operators. Let $\varphi(x)$ be the scale basis function of the interpolation function, and the interpolation operator is defined as follows: 


$$
I_{j} f=\sum_{k=0}^{2^{j}} f\left(x_{j k}\right) \varphi_{j k}, \quad x_{j k}=k 2^{-j} .
$$

For function $f(x) \in L^{2}(0,1)$, large enough $J$ is found to make $f_{J}(x) \in V_{J}$ to approximate $f(x)$. Let the coefficients of wavelet function and scale function be $\alpha_{j, k}$ and $\beta_{j_{0}, k}$, respectively, then

$$
f_{J}(x)=\sum_{k=0}^{2^{j_{0}}} \beta_{j_{0}, k} \varphi_{j_{0}, k}(x)+\sum_{j=j_{0}}^{J-1} \sum_{k=0}^{2^{j}-1} \alpha_{j, k} \psi_{j, k}(x),
$$

where $\beta_{j_{0}, k}=f\left(x_{j_{0}, k}\right)$ and $x_{j_{0}, k}$ is the characteristic point of the wavelet on the $j_{0}-$ th layer. And the wavelet coefficient $\alpha_{j, k}$ is defined as follows:

$$
\alpha_{j, k}=f\left(y_{j, k}\right)-Q_{j} f\left(y_{j, k}\right),
$$

where $y_{j, k}=x_{j+1,2 k+1}$ and $Q_{j}$ represents the wavelet interpolation operator of the $j_{0}-$ th layer. The calculation formula of the interpolated wavelet coefficients can be obtained by combining the above two formulas:

$$
\begin{aligned}
\alpha_{j, k}= & f\left(y_{j, k}\right)-\left[\sum_{k_{0}=0}^{2^{j_{0}}} f\left(x_{j_{0}, k_{0}}\right) \varphi_{j_{0}, k_{0}}\left(y_{j, k}\right)\right. \\
& \left.+\sum_{j_{1}=j_{0}}^{j-1} \sum_{k_{1}=0}^{2^{j_{1}}-1} \alpha_{j_{1}, k_{1}} \psi_{j_{1}, k_{1}}\left(y_{j, k}\right)\right] .
\end{aligned}
$$

The wavelet transform coefficient has an intuitive geometric meaning, which is the error between the interpolation result and the exact result at the same collocation point. It reflects the local regularity of the approximated function. By multiplying the matrix with the signal, the multiscale wavelet transform coefficients of the signal can be obtained at one time, which can be used to identify the salt-and-pepper noises in the slice images. Then, after multiscale interpolation wavelet reconstruction, salt-and-pepper noise is effectively removed. According to the interpolation wavelet transform theory [22], the wavelet coefficients can be written as

$$
\begin{aligned}
\alpha_{j}^{1}, k_{1}, k_{2}= & \varnothing\left(x_{2 k_{1}+1}^{j+1}, y_{2 k_{2}}^{j+1}\right)-I_{j} \varnothing\left(x_{2 k_{1}+1}^{j+1}, y_{2 k_{2}}^{j+1}\right) \\
= & \varnothing\left(x_{2 k_{1}+1}^{j+1}, y_{2 k_{2}}^{j+1}\right)-\left[\sum_{k_{01}=0}^{1} \sum_{k_{02}=0}^{1} \varnothing\left(x_{k_{01}}^{0}, y_{k_{02}}^{0}\right) w_{k_{01}, k_{02}}^{0}\right. \\
& \cdot\left(x_{2 k_{1}+1}^{j+1}, y_{2 k_{2}}^{j+1}\right)+\sum_{j_{1}=0}^{j-1} \sum_{k_{11}=0}^{2 j_{1}} \sum_{k_{12}=0}^{2 j_{1}}\left(\alpha_{j_{1}, k_{11}, k_{12}}^{1} \omega_{2 k_{1}+1}^{j_{1}+1}\right. \\
& \cdot\left(x_{2 k_{1}+1}^{j+1}, y_{2 k_{2}}^{j+1}\right)+\alpha_{j_{1}, k_{11}, k_{12}}^{2} w_{2 k_{11}, 2 k_{12}+1}^{j_{1}+1} \\
& \cdot\left(x_{2 k_{11}+1}^{j+1}, y_{2 k_{2}}^{j+1}\right)+\alpha_{j_{1}, k_{11}, k_{12}}^{3} w_{2 k_{11}, 2 k_{12}+1}^{j_{1}+1} \\
& \left.\left.\cdot\left(x_{2 k_{1}+1}^{j+1}, y_{2 k_{2}}^{j+1}\right)\right)\right]
\end{aligned}
$$

where $w_{k_{1}, k_{2}}^{j(m, n)}(x, y)$ denotes the multiscale wavelet function and the corresponding $m$ th and $n$th derivatives with respect to $x$ and $y$, respectively. $\left(x_{k_{1}}^{j}, y_{k_{2}}^{j}\right)$ is the position of the pixel after the discrete, and $I_{j}$ denotes the multilevel interpolation operator, where $j$ and $J$ are constants, which denote the wavelet scale number and the maximum of the scale number, respectively. The similar method can be used to obtain wavelet transform coefficients $\alpha_{j, k_{1}, k_{2}}^{2}$ and $\alpha_{j, k_{1}, k_{2}}^{3}$ at the points $\left(x_{k_{1}}^{j}, y_{k_{2}}^{j}\right)$.

After wavelet transform, the useful signals in the image are mainly retained in the small wavelet coefficients. However, the signals energy of salt-and-pepper noise mainly exists in the large wavelet coefficients. So, signals between the different size thresholds are preserved by choosing the appropriate threshold. Then, the salt-and-pepper noise can be effectively removed. The description of image detail feature pixels at different scales can be achieved by setting wavelet coefficient thresholds at different scales, which is

$$
\left|\alpha_{j, k_{1}, k_{2}}^{i}\right|<A_{j}^{1 / 2} \mathcal{E}, \quad i=1,2,3
$$

where $\varepsilon$ is the wavelet threshold at $j=0$ and $A_{j}$ is the scaling parameter, which is usually taken as $1 / 2^{j}$. During the iterative process in solving the nonlinear PDEs by means of the wavelet numerical method, most of the discrete points in which the wavelet transform coefficients are smaller than the threshold epsilon can be neglected, and this is helpful for improving the efficiency of the algorithm.

In order to obtain the multilevel interpolation operator, it is necessary to express the wavelet coefficients as a weighted sum of $u$ in all of the coefficient points in the $J$-level. So, the constraint operator $R_{k, n}^{j, J}$ is introduced. It can be seen from the meaning represented by interpolating wavelet operator. All the wavelet characteristic points on the $j$-layer can get the approximation function on the $j$-layer by interpolating the wavelet operator. The expression is as follows:

$$
f_{J}(x)=\sum_{n=0}^{2^{J}} Q_{J}(x) f\left(x_{J, n}\right) .
$$

The interpolation wavelet operator is as follows:

$$
Q_{J}=\sum_{k=0}^{2^{j} 0} R_{k, n}^{j_{0} J} \varphi_{j_{0}, k}(x)+\sum_{j=j_{0}}^{J-1} \sum_{k=0}^{2^{j}-1} C_{k, n}^{j, J} \psi_{j . k}(x),
$$

where $\quad C_{k, n}^{j, J}=R_{2 k+1, n}^{j+1, J}-\sum_{k_{0}=0}^{2^{j_{0}}{ }_{0}} R_{k_{0}, n}^{j_{0} J} \phi_{j_{0}, k_{0}}\left(x_{j+1,2 k+1}\right)-\sum_{j_{1}=j_{0}}^{j-1}$ $\sum_{k_{1}=0}^{2^{j_{1}}-1} C_{k_{1}, n}^{j_{1} J} \psi_{j_{1}, k_{1}}\left(x_{j+1,2 k+1}\right)$.

2.2. Texture Features and Collocation Points. The locust microslice image is the one that possesses some properties such as texture, smooth area, and boundary, and its structure is relatively complicated, as shown in Figure 1.

The block inside the number 1 rectangular box represents the image texture. The number 2 is a smooth area of the locust image, and the number 3 is the image edge. Noise is mostly distributed in high-frequency information. But edges and texture details are also high-frequency information. So, the edge structure and texture information will be removed at the same time of denoising. The collocation points are image characteristic description operator based on multiscale 


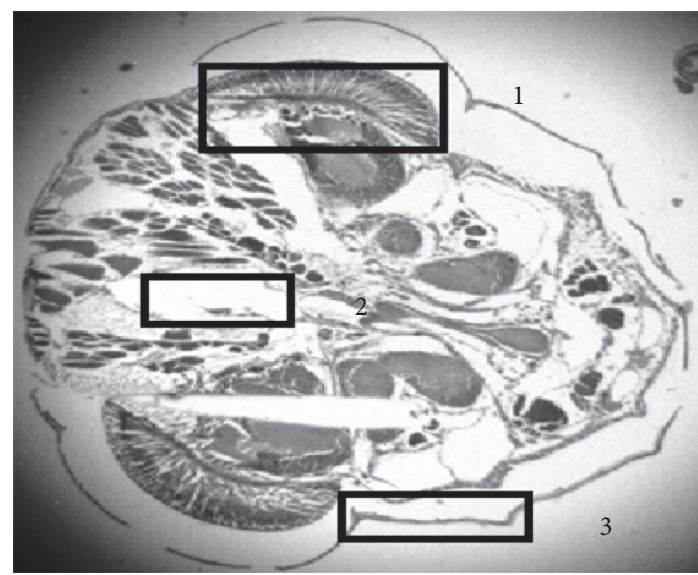

FIgURE 1: Locust microslice image. space. Shannon-cosine wavelet is applied to represent the slice images sparsely by adjusting the parameters adaptively based on the texture of the locust slice images. For contours with large gradient changes, more characteristic points are retained. However, in the detail texture of the image with small gradient change, the retained characteristic points are decreased; thereby, the denoising accuracy of the locust slice images is improved. Multiscale refinement can highlight the features of detail information in the image [23]. The collocation points for the locust slice image is obtained by the sparse interpolation operator, as shown in Figure 2.

Multiscale interpolation wavelet can be widely used in the field of image processing. The purpose of constructing the multilevel wavelet collocation method is to decrease the amount of the collocation points and then improve the efficiency of the algorithm. It can be seen from Figure 2 that the collocation points are adaptively collocationed by sparse interpolation wavelet, sparsely extracting points in smooth areas and densely extracting points in detailed texture areas. According to statistics, the total number of pixels in locust slice is 90000 , and the number of adaptive collocation points is 12319. Obviously, the computation complexity is decreased greatly compared with processing all pixels. With the image represented sparsely, the collocation points are far less during image processing to save space and improve efficiency.

\subsection{Sparse Representation and Noise Restoration.} Multiscale interpolation operators are used to process the internal structure of the images. As shown in Figure 3(b), salt-and-pepper noises with a density of $0.03 \mathrm{~dB}$ are added. The denoising results of the sparse interpolation are shown in Figure 3(c). It can be seen from Figure 3 that the sparse representation wavelet can identify and remove salt-andpepper noise through multiscale wavelet transform coefficients. The collocation points are adaptively captured by sparse interpolation wavelets, as shown in Figure 3(d). The total number of pixels in this image is 90000 , and the number of adaptive collocation points is 13764 . The efficiency is about $85 \%$ higher than the original efficiency. So, the multiscale interpolation wavelet can not only sparsely represent the image by the texture of the image but also

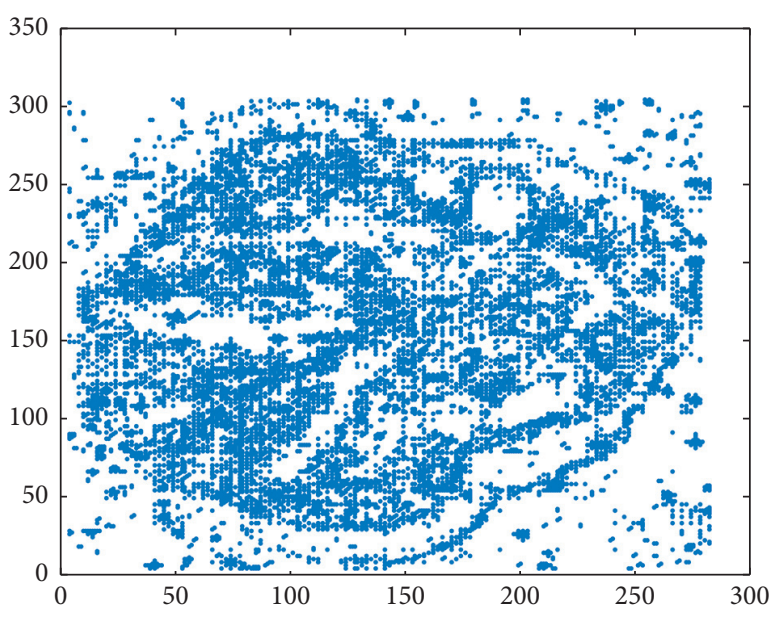

Figure 2: Sparse dot of locust slice image.

identify and remove the salt-and-pepper noise of highfrequency information.

The noise points usually distribute in high-frequency information, while effective information distributes in low-frequency information. Therefore, the noise point can be separated from the information point by setting threshold. The denoising effect of the image is affected by setting the size and number of thresholds. The wavelet transform coefficients are smaller than the threshold epsilon that can be neglected. In this paper, two thresholds are set to help improve the efficiency of the algorithm. With the increasing number of iterations, part of the texture at the edge of the images are treated as noise points, which decreases denoising performance. The effect of the proposed algorithm to remove salt-and-pepper noise is shown in Figure 4. The result shows that the number of iterations should not be too large when removing salt-and-pepper noise.

\section{Sparse Wavelet Precise Integration Method (SWPIM)}

3.1. Denoising Model and Problem Analysis of Nonlinear PDE. Perona-Malik adopted different diffusion speeds in the interior and edge of the feature area to achieve the effect of protecting the image boundary [24]. Perona-Malik edgepreserving smoothing model is defined as follows:

$$
\left\{\begin{array}{l}
\frac{\partial u(x, y, t)}{u(x, y, 0)}=\operatorname{div}(c(|\nabla u|) \nabla u), \\
u(x, y, 0)=f(x, y),
\end{array}\right.
$$

where $(x, y)$ denotes the pixel point position and $f(x, y)$ denotes the image to process. $t$ is the time parameter. $u(x, y, t)$ denotes the image after processing, and $u(x, y, 0)$ denotes the initial value. div is the diffusion operator. $\nabla$ denotes the gradient of the image, and $c(|\nabla u|)$ represents the diffusion coefficient. By substituting equations (10) and (11) into equation (12), the nonlinear PDE can be discretized into nonlinear ordinary differential equations. The ordinary differential equations are solved by precise integration. The discrete form of PM denoising equation is defined as follows: 


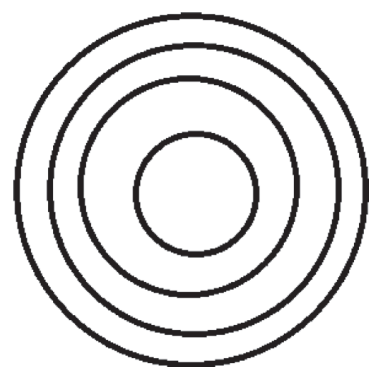

(a)

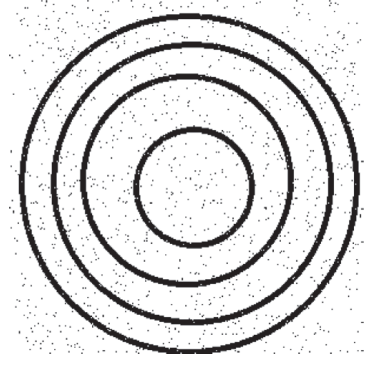

(b)

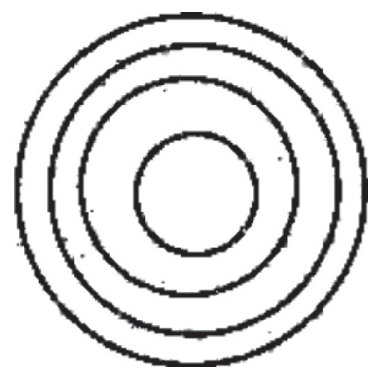

(c)

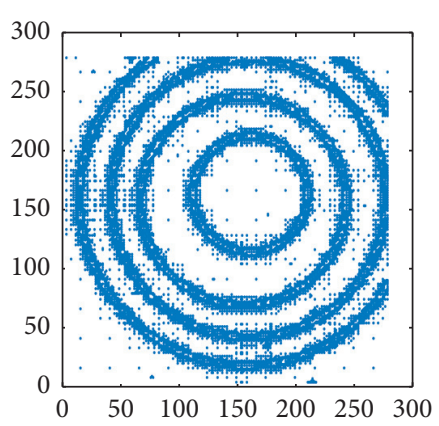

(d)

FIGURE 3: Sparse interpolation operator denoising: (a) original image; (b) images with salt-and-pepper; (c) sparse interpolation wavelet; (d) collocation points.

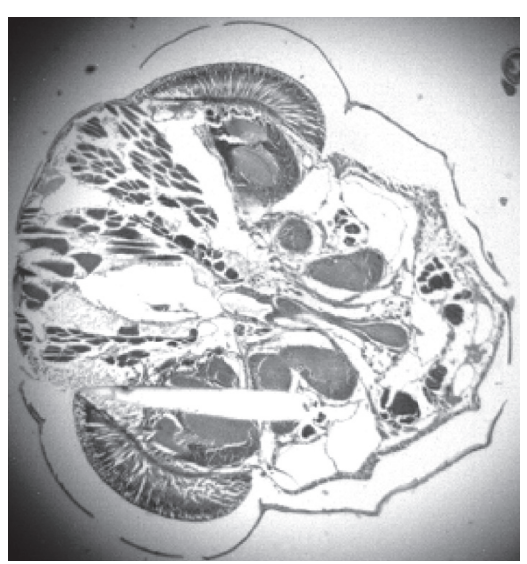

(a)

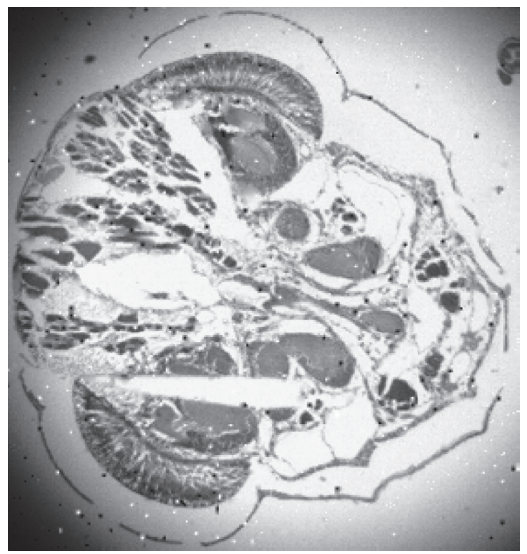

(d)

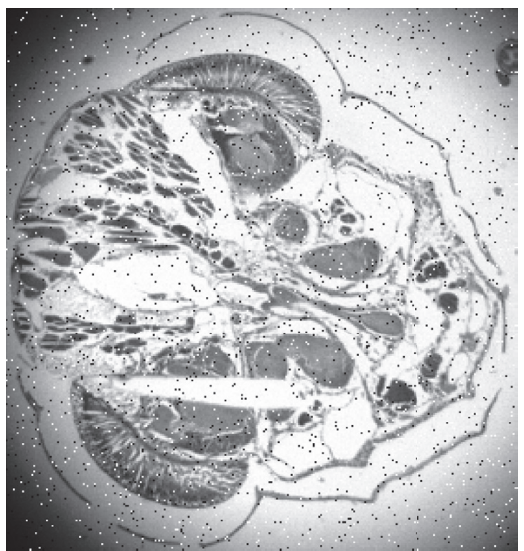

(b)

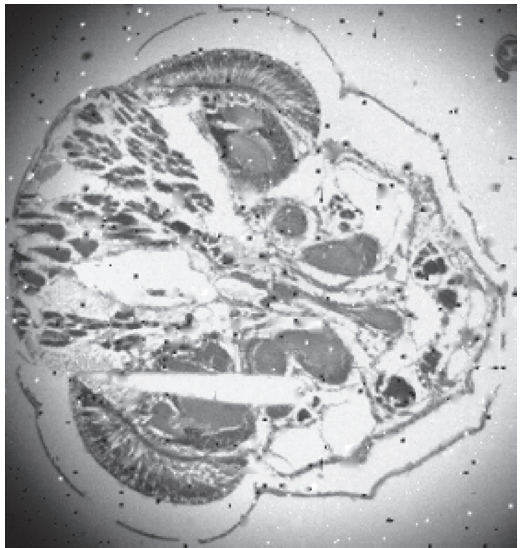

(e)

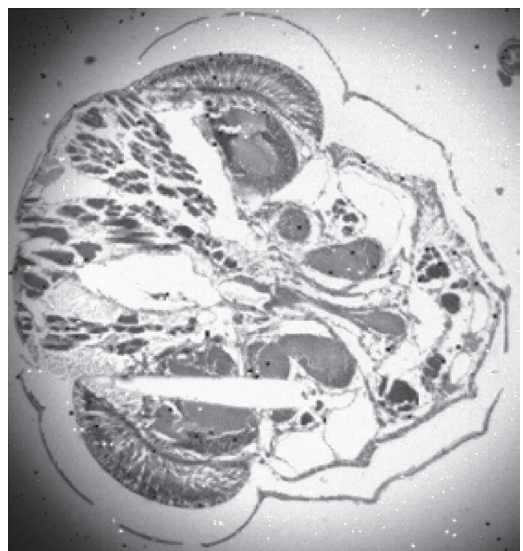

(c)

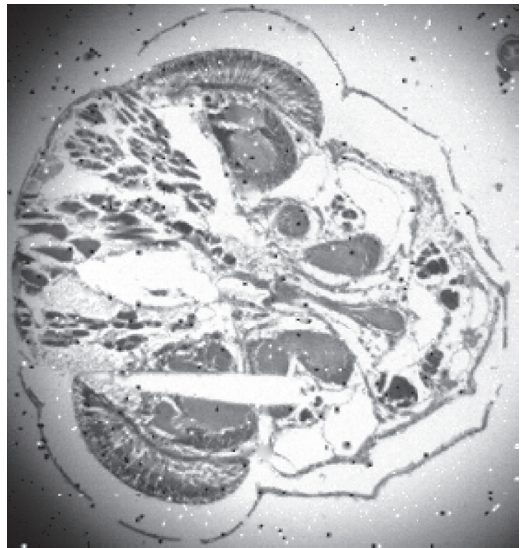

(f)

FIGURE 4: Increase in the number of iterations: (a) original image; (b) images with salt-and-pepper noise; (c) image with 1 iteration; (d) image with 2 iterations; (e) image with 3 iterations; (f) image with 4 iterations.

$$
\begin{aligned}
\frac{\partial u(x, y, t)}{\partial t}= & \frac{\partial[c(x, y, t)(\partial u(x, y, t) / \partial x)]}{\partial x}+\frac{\partial[c(x, y, t)(\partial u(x, y, t) / \partial y)]}{\partial y} \\
\approx & \frac{1}{(\Delta x)^{2}}\left[c\left(x+\frac{\Delta x}{2}, y, t\right)(u(x+\Delta x, y, t)-u(x, y, t))-c\left(x-\frac{\Delta x}{2}, y, t\right)(u(x, y, t)-u(x-\Delta x, y, t))\right] \\
& +\frac{1}{(\Delta y)^{2}}\left[c\left(x, y+\frac{\Delta y}{2}, t\right)(u(x, y+\Delta y, t)-u(x, y, t))-c\left(x, y-\frac{\Delta y}{2}, t\right)(u(x, y, t)-u(x, y-\Delta y, t))\right] .
\end{aligned}
$$


Let the right side of formula (13) be noted as $\varphi$. Then, formula (13) can be written as follows:

$$
u(x, y, t+\Delta t) \approx u(x, y, t)+\Delta t \varphi .
$$

Substituting the sparse representation of the slice images into the Perona-Malik model which is the famous edgepreserving smoothing model for removing the Gaussian noises of the biomedical images, a system of nonlinear differential equations is obtained. A semianalytical method on solving the system of the nonlinear differential equations is constructed based on the sparse representation of the slice images, which is named SWPIM. With the application of $\mathrm{PDE}$ in image processing, the wavelet precise integration method has also been widely used in image processing [25-27]. The essence of precise integration method is the solution of matrix, which is simple and has high calculation accuracy. As the calculation process is almost unlimited by the time step, the calculation efficiency is greatly improved. Perona-Malik introduced the following two diffusion coefficients within the model studied $[28,29]$ :

$$
\begin{gathered}
c(|\nabla u|)=\frac{1}{1+(\nabla u / k)^{2}}, \\
\text { or } c(|\nabla u|)=\exp \left[-\left(\frac{\nabla u}{k}\right)^{2}\right],
\end{gathered}
$$

where $k$ is used to control the degree of diffusion. With the increasing number of iterations, the gradient value of the image is smaller and smaller relative to $k$. In order to ensure the relative proportion between $\nabla u$ and $k$ to prevent the edges of the image from being smoothed, it is unreasonable for $k$ to be set as a constant. In the PM model, the image contour and noise are identified by the parameter $k$. In order to accurately recognize Gaussian noise and image contour of the image, a method of adaptively selecting the parameter $k$ is proposed in this paper. Linear relationship between image contrast and $k$ value is expressed with the following formula:

$$
k=a x+b,
$$

where $x$ denotes the contrast of the image and the $a$ and $b$ are constants.

The position of the PM model with a larger image gradient value is regarded as the image contour. When the diffusion coefficient decreases, the smoothing speed of the diffusion equation at the image contour will become slower. Since the difference value at the noise is smaller than the difference value at the contour, the attenuation speed will increase. That is, for the same number of iterations, the edge will be protected by PDE, and the salt-and-pepper noise will not be filtered out. There are many ways to solve partial differential equations, and the most typical method is the difference method [30]. This method uses the flat function to describe image, approximately the surface function. But, it is easy to cause artifacts phenomenon, affecting the accuracy of image denoising. Wavelet function has both smooth and compactly supported characteristics. Besides, performance of multiscale analysis can be used to construct the multiscale adaptive interpolation operator for solving nonlinear partial differential equations [31].
3.2. Determination of Parameter $k$. As we all know, human vision is very sensitive to the contour and texture of the object. In the process of denoising, the edge texture of the images is usually treated as noise points, which makes the images blurred. So, it is necessary to effectively identify the boundary information by adjusting $k$ so that the boundary parts are not treated as noise points. The determination of $k$ value is closely related to the image contrast. The enhancement of image contrast will increase the gradient value of the contour pixels of the object in the image. $k$ for identifying contours and noise thresholds should also be adjusted accordingly. Based on this, a method for adaptively calculating the parameter $k$ is designed in this paper. The contrast of the locust slice images is calculated to determine the boundary texture of the image. At the same time, the Gaussian noise can be removed well by adaptively adjusting the $k$ value. For the locust slice images, the relationship formula between $k$ value and image contrast constructed in this paper is defined as follows:

$$
\begin{cases}40, & x_{m n} \leq 100, \\ k=-\frac{7}{30} x_{m n}+\frac{190}{3}, & 100<x_{m n}<250 \\ 5, & x_{m n} \geq 250\end{cases}
$$

where $x_{m n}$ denotes image contrast. Image contrast means difference of brightness in the image or the magnitude of gray contrast of the image [32]. The larger the range of difference, the greater the contrast. On the contrary, the smaller the difference range, the smaller the contrast. Early image contrast algorithms only considered image enhancement, not the measurement of image contrast. In this paper, this factor can be taken into account by using a contour detection operator in a window of appropriate size. Specifically, with the current pixel $(m, n)$ as the center, the number of pixels in the window should be odd rows and odd columns. The window should be large enough to ensure that noise is filtered out. At the same time, the window should be small enough to ensure that the visible details in the image can be preserved. Then, the boundary value $\Delta_{i j}$ of each pixel $(i, j)$ is calculated in each window $W_{m n}$. The boundary value is used to determine whether it is a weight of the boundary value. If the boundary value is 0 , it cannot be determined as a boundary. If the boundary value is equal to 1 (after normalization), the pixel must be a point on the boundary. Laplacian operator and Sobel operator can be used to calculate the boundary value $\Delta_{i j}$. These operators are not sensitive to noise compared with other operators. In this paper, the Laplacian operator is used to calculate the boundary weight $\Delta_{i j}$ :

$$
\Delta_{i j}=\left|X_{i j}-\bar{X}\right|
$$

where $\bar{X}$ denotes the average gray value of eight neighboring pixels of pixel $(i, j)$. And $X_{i, j}$ is set to the gray value at pixel $(i, j)$. In each window $W_{m n}$, the average boundary gray value $\bar{E}_{m n}$ (that is, the average gray value of the point at the target 
boundary) is calculated. The gray value of the target boundary can be obtained by calculating the weighted average of the gray value of the pixel. The definition of the average gray value of the boundary is defined as follows:

$$
\bar{E}_{m n}=\frac{\left(\sum_{(i, j) \in W_{m n}} \Delta_{i j} \bullet X_{i j}\right)}{\left(\sum_{(i, j) \in W_{m n}} \Delta_{i j}\right)} .
$$

In this paper, a large window $W_{m n}$ is used to ensure that these operators are not sensitive to noise. The image contrast $x_{m n}$ at the center pixel $(m, n)$ of the window is

$$
x_{m n}=\frac{\left|X_{m n}-\bar{E}_{m n}\right|}{\left|X_{m n}+\bar{E}_{m n}\right|} \text {. }
$$

Figure 5 shows locust slice images with different contrasts. Figure 6 shows that Gaussian noise with a standard deviation of 0.002 is added to the locust slice image with different contrasts. Figure 7 shows that the locust slice images with Gaussian noise are denoised by the proposed algorithm. Table 1 shows the $k$, PSNR, and SSIM values corresponding to the optimal denoising effect of locust slice images with different contrasts. From Figures 5-7 and Table 1 , it can be seen that the corresponding $k$ values in the denoising process are different for locust slice images with different contrasts. And the effect of using the proposed algorithm for processing is also different. The image contrast is linearly correlated with the adaptive $k$ value within a certain range. The experimental results show that the boundary information can be effectively identified by the adaptive $k$ value so that the boundary part is not treated as a noise point. It provides convenience for subsequent denoising work and makes the image denoising effect more significant.

\subsection{Sparse Interpolation Wavelet Precise Integration Method.} A novel denoising method for removing mixed noise from locust slice images is proposed by means of Shannon-cosine wavelet and the nonlinear partial differential equation variational model for the image processing. This method includes two parts that are the sparse representation of the slice images and the novel numerical algorithm for solving the variation model on image denoising based on the sparse representation. In the first part, a parametric Shannon-cosine wavelet function is introduced to construct the multiscale wavelet transform matrix, which is applied to represent the slice images sparsely by adjusting the parameters adaptively based on the texture of the locust slice images. By multiplying the matrix with the signal, the multiscale wavelet transform coefficients of the signal can be obtained at one time, which can be used to identify the saltand-pepper noises in the slice images. This ensures that the salt-and-pepper noises points are kept away from the sparse representation of the slice images. In the second part, a semianalytical method on solving the system of the nonlinear differential equations is constructed based on the sparse representation of the slice images, which is named SWPIM. Substituting the sparse representation of the slice images into the Perona-Malik model which is the famous edge-preserving smoothing model for removing the Gaussian noises of the biomedical images, a system of nonlinear differential equations is obtained, whose scale is far smaller than one obtained by the difference method. Figure 8 is the flow chart of the proposed algorithm.

\section{Application of Coupling Algorithm in Biological Slice Images}

According to the effect of noise on the image, the noise is divided into two categories: additive noise and multiplicative noise. This paper focuses on additive noise, and the mixed noise is considered to denoise. The mixed noise includes both Gaussian noise and salt-and-pepper noise. In order to verify the denoising performance of the proposed algorithm on locust slice images, the problem of image denoising with single noise (Gaussian noise or salt-and-pepper noise) is also considered. Then, these noise test images are restored by the proposed algorithm. The mathematical model of the noise image is as follows:

$$
y(x)=y_{0}(x)+v(x), \quad x \in \Omega,
$$

where $y_{0}(x)$ is the original image and $y(x)$ is the image with noise $v(x), v(x)$ is the mixed noise. $x$ is the set of pixels. The density of salt-and-pepper noise pixels in the image is denoted by sp, while the standard deviation of the Gaussian noise is denoted by $\sigma$.

This article uses Matlab for simulation experiments. In order to demonstrate the effectiveness of the proposed algorithm, it is compared with the representative denoising methods. Locust slice images with different image characteristics are used as test images. In order to evaluate the performance, the denoising algorithm of this paper and other denoising algorithms are compared in terms of objective indicators and visual effects.

\subsection{Evaluation and Analysis of Objective Indicators}

4.1.1. Image Quality Evaluation Standard. The performance of image restoration results is quantitatively evaluated by two measures, PSNR (peak signal-to-noise ratio) and SSIM (structural similarity index). The higher the value of PSNR is, the better the quality of the restored image is. PSNR can be expressed as

$$
\begin{aligned}
\operatorname{PSNR} & =10 \log _{10}\left(\frac{255^{2}}{\mathrm{MSE}}\right), \\
\mathrm{MSE} & =\frac{1}{M N} \sum_{i=1}^{M} \sum_{j=1}^{N}[S(i, j)-\widehat{S}(i, j)]^{2},
\end{aligned}
$$

where $S(i, j)$ and $\widehat{S}(i, j)$ denote the original pixel and the restored pixel, respectively. $M$ and $N$ are the sizes of an image for the width and the height, respectively. The MSE denotes the mean square error between the original noisefree image and the restored image. The SSIM denotes the structural similarity between the original noise-free image and the restored image which is given by 


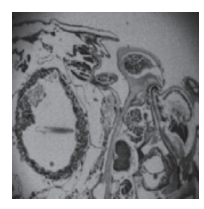

(a)

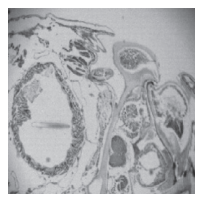

(h)

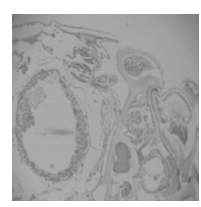

(b)

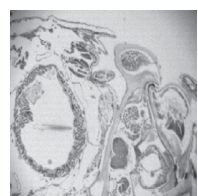

(i)

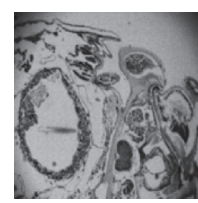

(c)

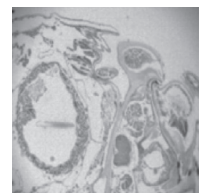

(j)

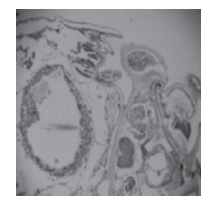

(d)

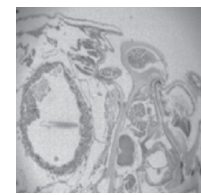

(k)

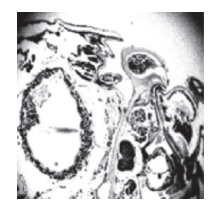

(e)

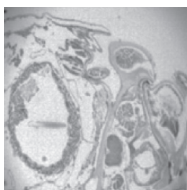

(1)

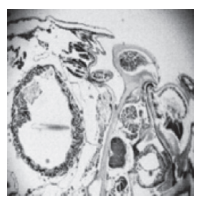

(f)

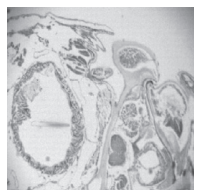

(m)

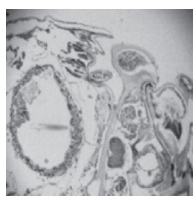

(g)

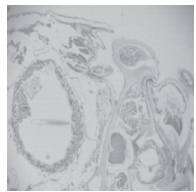

(n)

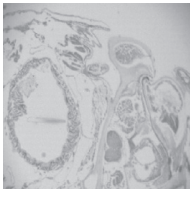

(o)

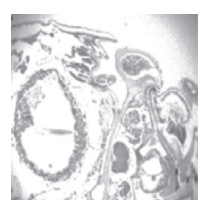

(p)

Figure 5: Original image with different image contrasts: (a) $x_{m n}=100.84$; (b) $x_{m n}=120.87$; (c) $x_{m n}=132.05$; (d) $x_{m n}=140.77$; (e) $x_{m n}=158.51$; (f) $x_{m n}=166.86$; (g) $x_{m n}=176.79$; (h) $x_{m n}=188.30 ;$ (i) $x_{m n}=200.37$; (j) $x_{m n}=210.01$; (k) $x_{m n}=213.34$; (l) $x_{m n}=222.19$; (m) $x_{m n}=226.18$; (n) $x_{m n}=238.59$; (o) $x_{m n}=241.13$; (p) $x_{m n}=244.45$.

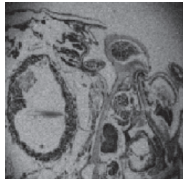

(a)

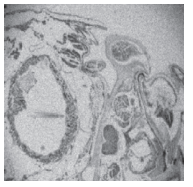

(h)

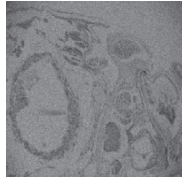

(b)

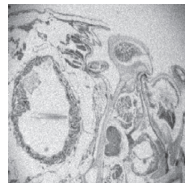

(i)

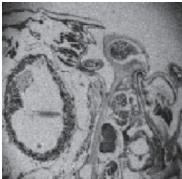

(c)

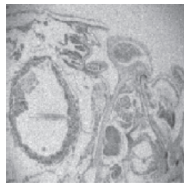

(j)

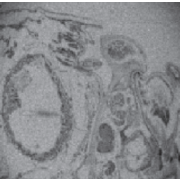

(d)

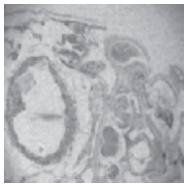

(k)

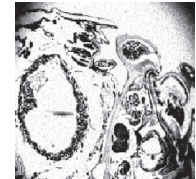

(e)

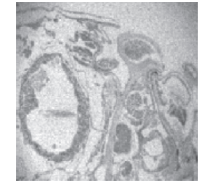

(1)

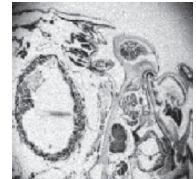

(f)

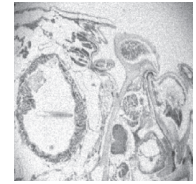

(m)

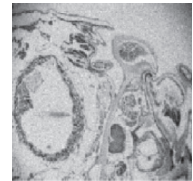

(g)

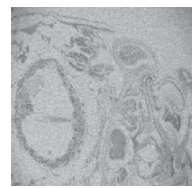

(n)

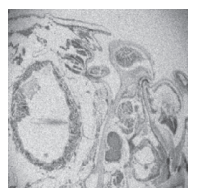

(o)

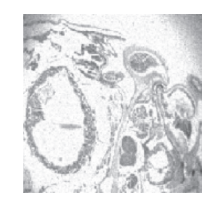

(p)

Figure 6: Images with different contrasts after adding the same standard deviation of Gaussian noise.

$$
\operatorname{SSIM}=\frac{\left(2 \mu_{x} \mu_{y}+c_{1}\right)\left(2 \sigma_{x y}+c_{2}\right)}{\left(\mu_{x}^{2}+\mu_{y}^{2}+c_{1}\right)\left(\sigma_{x}^{2}+\sigma_{y}^{2}+c_{2}\right)},
$$

where $\mu_{x}$ and $\mu_{y}$ are the means of the input image and the restored image, respectively. $\sigma_{x y}$ is the covariance of the input image and the restored image. $\sigma_{x}^{2}$ and $\sigma_{y}^{2}$ are the variances of the input image and the restored image, respectively. $c_{1}$ and $c_{2}$ are the constants, which can be calculated according to the paper. The higher value of SSIM means that the structures in the restored image can be preserved more completely.
4.1.2. Objective Evaluation of Image Denoising Performance Contaminated by Salt-and-Pepper Noise. In the first set of experiments, the problem of image denoising is considered with salt-and-pepper noise. The image in Figure 1 is used as the test image. It is added to the salt-and-pepper noise with different degrees: the density of salt-and-pepper noise varies with $0.01,0.02,0.03,0.04$, and 0.05 . The proposed algorithm is compared with representative denoising methods including the improved median filtering (IMED), mean filtering (MEA), Wiener filtering (WI), wavelet transform (WA), improved nonlocal mean filtering (INLM), bilateral filtering (BI), median and Wiener combination (MWI), 


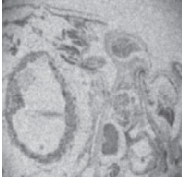

(a)

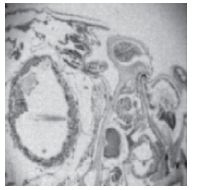

(h)

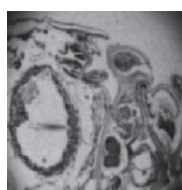

(b)

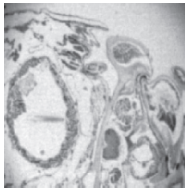

(i)

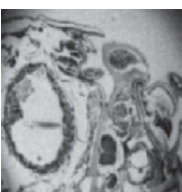

(c)

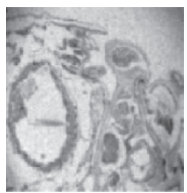

(j)

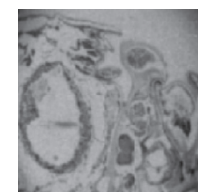

(d)

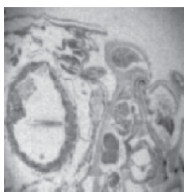

(k)

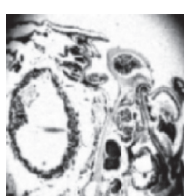

(e)

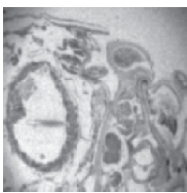

(1)

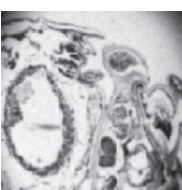

(f)

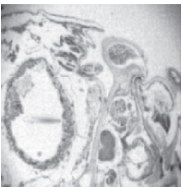

(m)

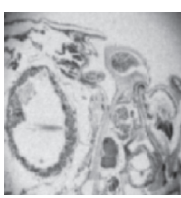

(g)

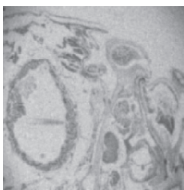

(n)

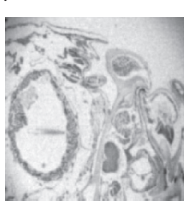

(o)

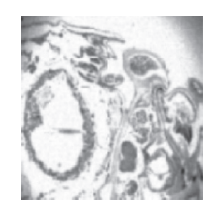

(p)

FIgURE 7: Images after denoising with different contrasts.

TABle 1: $k$ value corresponding to different image contrasts and denoising effect.

\begin{tabular}{lcccc}
\hline Image number & Contrast & PSNR & SSIM & $k$ \\
\hline 1 & 100.84 & 24.628 & 0.781 & 38 \\
2 & 120.87 & 31.804 & 0.821 & 35 \\
3 & 132.05 & 23.874 & 0.780 & 31 \\
4 & 140.78 & 28.086 & 0.814 & 31.3 \\
5 & 158.51 & 17.740 & 0.769 & 30 \\
6 & 166.86 & 21.543 & 0.780 & 28.2 \\
7 & 176.79 & 25.404 & 0.795 & 24 \\
8 & 188.30 & 30.450 & 0.827 & 19.7 \\
9 & 200.38 & 27.114 & 0.816 & 15 \\
10 & 210.02 & 28.019 & 0.781 & 12.3 \\
11 & 213.34 & 28.759 & 0.798 & 12.3 \\
12 & 222.19 & 27.731 & 0.782 & 11.4 \\
13 & 226.18 & 26.939 & 0.813 & 10 \\
14 & 238.59 & 31.225 & 0.852 & 7 \\
15 & 241.13 & 27.972 & 0.823 & 5 \\
16 & 244.46 & 25.242 & 0.799 & 5 \\
\hline
\end{tabular}

median and wavelet combination (MWA), and median and Gauss combination (MG). The quantitative indicators PSNR and SSIM data are shown in Tables 2 and 3, respectively. Table 2 presents the performance comparisons for the different denoising algorithms in terms of PSNR value. Table 3 presents the performance comparisons for the different denoising algorithms in terms of SSIM value.

As shown in Figure 9, the changes in the PSNR and SSIM evaluation indicators in Tables 2 and 3 are vividly and intuitively reflected through line charts. Figure 9 shows how PSNR and SSIM varies for the denoised images as the saltand-pepper noise sp varies from 0 to 0.05 . It can be seen that, in the case of low noise corruption (sp less than 0.03), all the denoising algorithms can perform well.

It can be seen from Tables 2 and 3 that the proposed algorithm shows a good ability to deal with salt-and-pepper noise. The PSNR of the image restoration results do not decrease significantly. With the increase in salt-and-pepper noise from $0.01 \mathrm{~dB}$ to $0.03 \mathrm{~dB}$, the PSNR value of the proposed denoising algorithm has always been the largest, and the maximum value is $29.1610 \mathrm{~dB}$. The proposed algorithm reveals the superior denoising performance to the median filtering. The SSIM value of the proposed algorithm is also the largest. The similarity of the image processed using the proposed algorithm to the original image reaches 0.9612 . It means that the proposed algorithm has a stronger capability than other compared denoising algorithm for preserving image structures. As to the cases of heavy noise corruptions (noise density higher than 0.03), although the proposed algorithm's recovery results are not as good as the median filtering, the PSNR values are larger than other algorithms. Hence, Tables 2 and 3 indicate that the proposed denoising algorithm is able to more effectively remove salt-and-pepper noise than other compared denoising algorithm.

4.1.3. Objective Evaluation of Image Denoising Performance Contaminated by Gaussian Noise. In the second set of experiments, the problem of image denoising with Gaussian noise is considered. The image in Figure 10(a) is used as the test image. It is added to the Gaussian noise with different degrees: the standard deviation of Gaussian noise varies with $0.001,0.002,0.003,0.004$, and 0.005 . The proposed algorithm is compared with representative denoising methods including the improved median filtering (IMED), mean filtering (MEA), Wiener filtering (WI), wavelet transform (WA), bilateral filtering (BI), improved nonlocal mean filtering (INLM), and median and Wiener combination (MWI). The quantitative indicators PSNR and SSIM data are shown in Tables 4 and 5, respectively. Table 4 presents the performance comparisons for the different denoising algorithms in terms of PSNR value. Table 5 presents the performance comparisons for the different denoising algorithms in terms of SSIM value. 


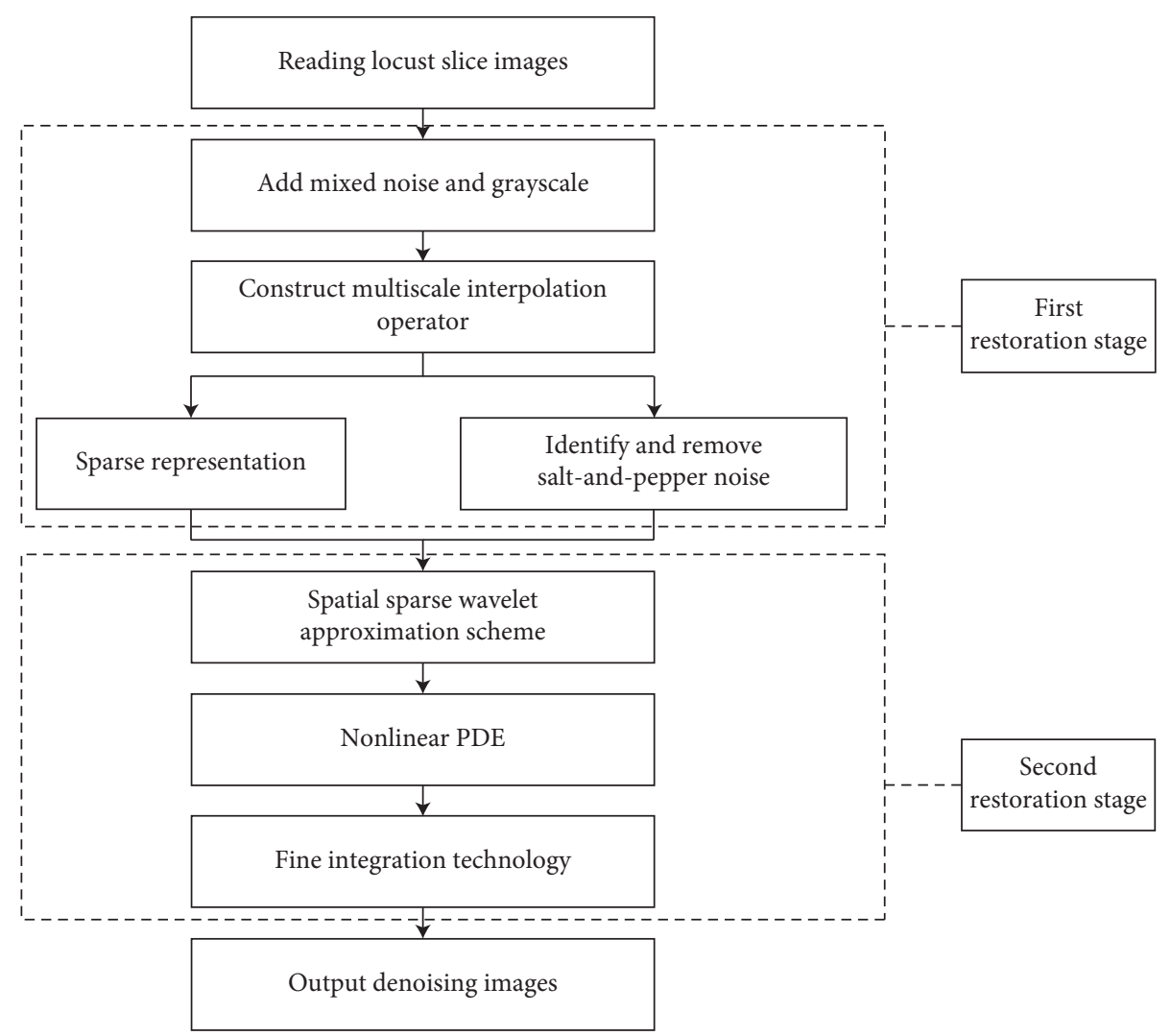

FIGURE 8: Flow chart of Shannon-cosine wavelet fine integration denoising algorithm.

TABLE 2: Comparison with other salt-and-pepper noise removal methods, showing PSNR values for varying amounts of salt-and-pepper noise.

\begin{tabular}{lcccccccccc}
\hline sp $(\mathrm{dB})$ & IMED & MEA & WI & WA & BI & INLM & MWI & MWA & MG & Proposed \\
\hline 0.01 & 26.8004 & 24.397 & 24.270 & 24.650 & 23.868 & 24.5128 & 25.592 & 24.164 & 23.729 & $\mathbf{2 9 . 1 6 1}$ \\
0.02 & 26.6843 & 23.839 & 22.117 & 22.829 & 21.236 & 21.6082 & 25.526 & 24.135 & 23.446 & $\mathbf{2 7 . 5 6 3}$ \\
0.03 & 26.4995 & 23.320 & 20.738 & 21.485 & 19.397 & 19.7028 & 25.414 & 23.839 & 23.129 & $\mathbf{2 6 . 5 9 2}$ \\
0.04 & $\mathbf{2 5 . 4 4 5 3}$ & 22.921 & 20.043 & 20.673 & 18.437 & 18.531 & 25.383 & 23.837 & 22.840 & 25.417 \\
0.05 & $\mathbf{2 6 . 2 9 0 9}$ & 22.463 & 19.367 & 19.965 & 17.405 & 17.6494 & 25.288 & 23.602 & 22.655 & 25.316 \\
\hline
\end{tabular}

TABLE 3: Comparison with other salt-and-pepper noise removal methods, showing SSIM values for varying amounts of salt-and-pepper noise.

\begin{tabular}{lccccccccccc}
\hline sp $(\mathrm{dB})$ & IMED & MEA & WI & WA & BI & INLM & MWI & MWA & MG & Proposed \\
\hline 0.01 & 0.879 & 0.779 & 0.784 & 0.775 & 0.781 & 0.8565 & 0.837 & 0.779 & 0.770 & $\mathbf{0 . 9 6 1}$ \\
0.02 & 0.877 & 0.738 & 0.697 & 0.700 & 0.679 & 0.7514 & 0.836 & 0.778 & 0.738 & $\mathbf{0 . 9 4 0}$ \\
0.03 & 0.874 & 0.701 & 0.628 & 0.631 & 0.594 & 0.6626 & 0.833 & 0.767 & 0.716 \\
0.04 & 0.873 & 0.675 & 0.592 & 0.593 & 0.540 & 0.5928 & 0.833 & 0.767 & 0.690 & $\mathbf{0 . 9 2 0}$ \\
0.05 & 0.870 & 0.641 & 0.543 & 0.542 & 0.478 & 0.5405 & 0.831 & 0.757 & 0.672 & $\mathbf{0 . 8 9 2}$ \\
\hline
\end{tabular}

In order to intuitively reflect the changes in the PSNR and SSIM evaluation indicators in Tables 4 and 5, data comparison line charts are given as shown in Figure 11. It shows how PSNR and SSIM varies for the denoised images as the Gaussian noise $\sigma$ varies from 0.001 to 0.005 .

The higher the value of PSNR is, the better the quality of the restored image is. It can be seen from Table 4 and Figure 11(a) that the proposed algorithm usually obtains the largest PSNR value, and the maximum value is $31.9292 \mathrm{~dB}$. In the same noise corruption, the proposed algorithm can perform better than other algorithms. In the case of low noise corruption ( $\sigma$ less than 0.005), all of the difference values between the proposed algorithm and the WI are greater than $1 \mathrm{~dB}$ and those between the proposed algorithm and the IMED are greater than $2 \mathrm{~dB}$. At the same time, the PSNR value of INLM restoration image is about $3 \mathrm{~dB}$ higher 


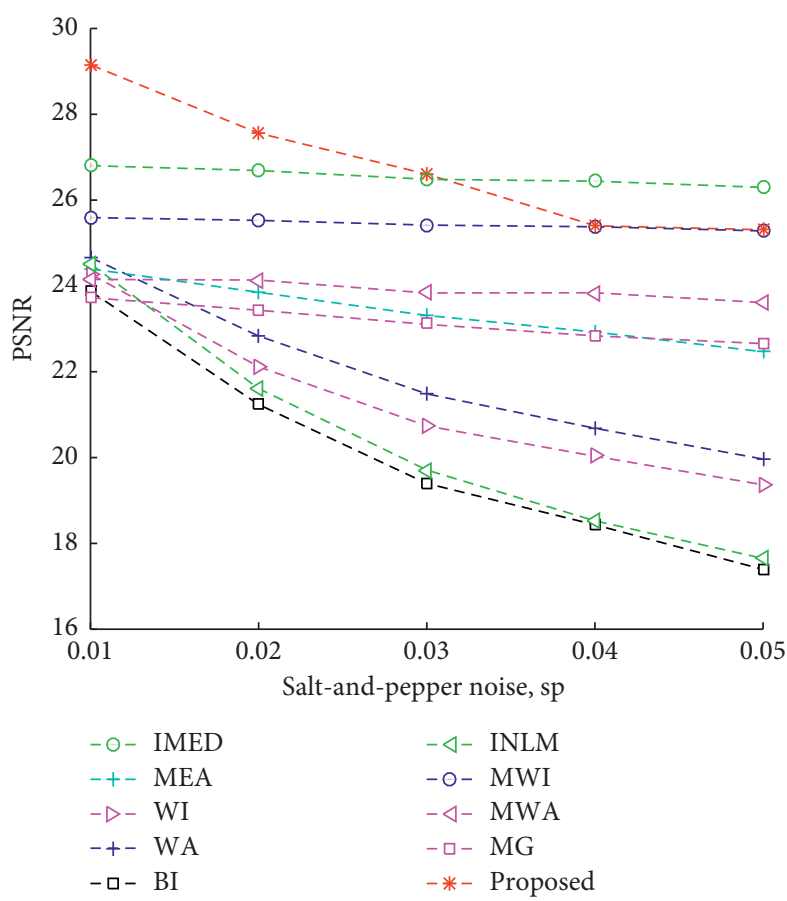

(a)

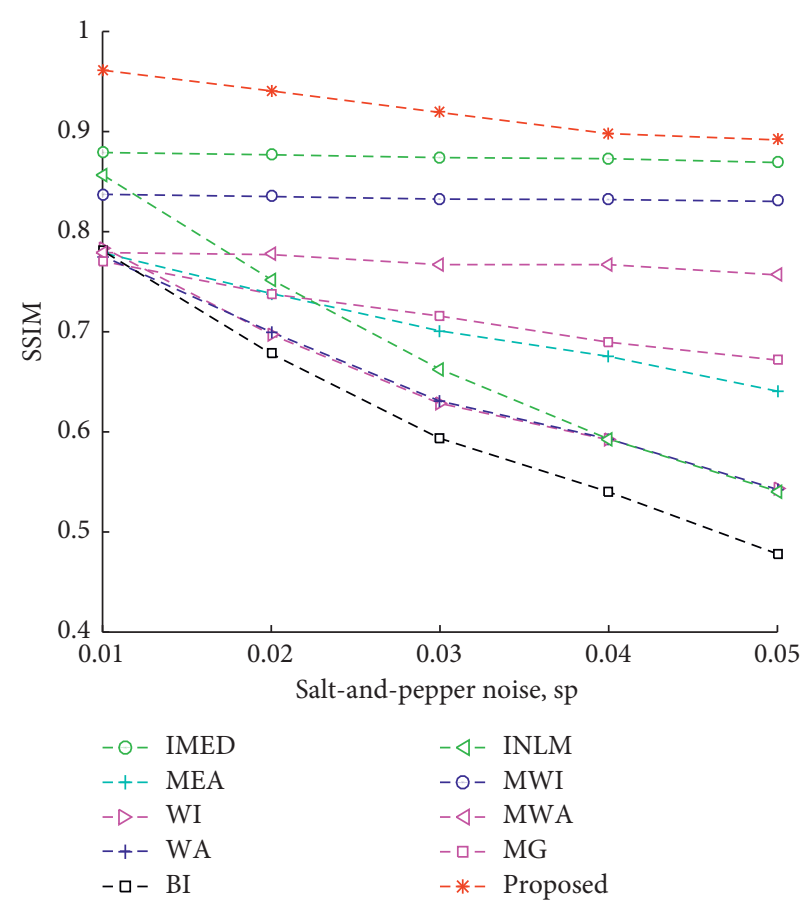

(b)

FIGURE 9: Variation in (a) PSNR and (b) SSIM with varying amounts of salt-and-pepper noise.

than that of the proposed algorithm. From the quantitative comparison as shown in Table 5 and Figure 11(b), we can see that the SSIM of our image recovery results is always the highest in Gaussian low-noise environment, and the maximum value is 0.8966 . As to the cases of heavy noise corruptions, the SSIM value of INLM restored image is about 0.2 higher than that of the proposed algorithm. So, in Gaussian noise environments, the proposed algorithm reveals the superior denoising performance than the other algorithms. In other words, the structures in the restoration results of the proposed algorithm can be preserved more completely than other algorithms. The proposed algorithm can achieve the larger values of PSNR and SSIM than other algorithms, which can indicate that the proposed algorithm performs better than the other algorithms in image denoising and detail preservation.

\subsubsection{Objective Evaluation of Image Denoising Performance} Contaminated by Mixed Noise. In the third set of experiments, image denoising with mixed noise is considered, which includes Gaussian noise and salt-and-pepper noise. Further quantitative results for the all algorithms are obtained using input images generated by adding salt-andpepper noise with different levels $(\mathrm{sp}=0.01,0.02,0.03)$ mixed with Gaussian noise with different levels $(\sigma=0.001$, $0.002,0.003,0.004,0.005)$. The locust slice image in Figure $12(\mathrm{a})$ is used as the test image. The proposed algorithm is compared with representative denoising methods including the improved median filtering (IMED), mean filtering (MEA), Wiener filtering (WI), wavelet transform (WA), bilateral filtering (BI), improved nonlocal mean filtering (INLM), median and Wiener combination (MWI), and median and wavelet combination (MWA). Tables 6 and 7 present the quantitative comparisons for the various algorithms in terms of the PSNR value and the SSIM value, respectively.

In order to visually illustrate performance, Figure 13 shows the trends of PSNR evaluation indicators for different mixed noise intensities. Figure 13(a) shows how PSNR of denoised images varies as the standard deviation of the Gaussian noise $\sigma$ varies from 0.001 to 0.005 with a fixed saltand-pepper noise density $\mathrm{sp}=0.01$. Figure 13 (b) shows how PSNR varies for the denoised images as the sp varies from 0 to 0.03 with fixed Gaussian noise $\sigma=0.001$.

From Figure 13(b) and Table 7, we can see that the ability of the proposed algorithm is better to process salt-andpepper noise. In low salt-and-pepper noise environments, as the standard deviation of Gaussian noise increases from 0.001 to 0.005 , the proposed algorithm is superior to other algorithms. And compared to other algorithms, the proposed algorithm can obtain the higher value of PSNR in the cases of various noise densities. The difference between PSNR value of MWI restored image and SSIM value of MEA is about $1 \mathrm{~dB}$. The IMED and proposed algorithm denoising performance are almost similar. It can be seen from Table 7 that the SSIM values of the restoration results of the MEA, WI, and BI are significantly less than the MWI, IMED, INLM, and the proposed algorithm under the case of various noise densities. Compared with other algorithms, this proposed algorithm can achieve larger PSNR and SSIM values in a low noise densities environment. The proposed algorithm reveals the superior denoising performance than the IMED, MEA, INLM, and MWI. It means that the 


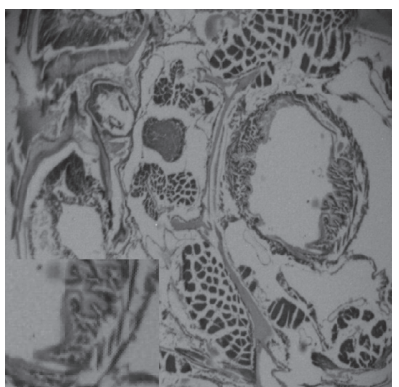

(a)

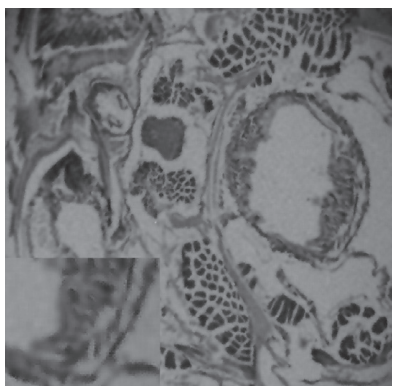

(e)

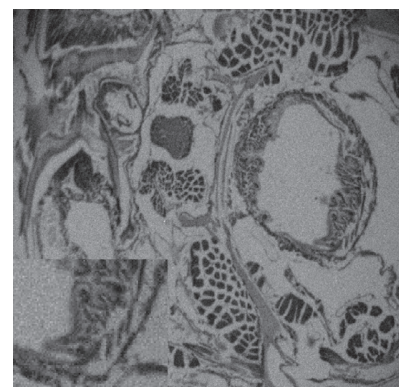

(b)

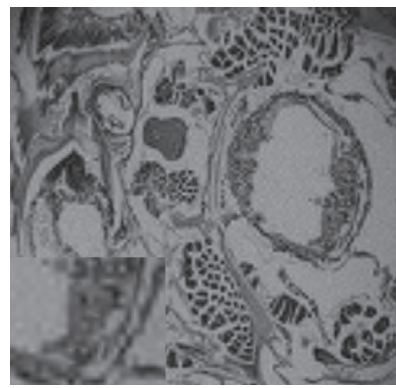

(f)

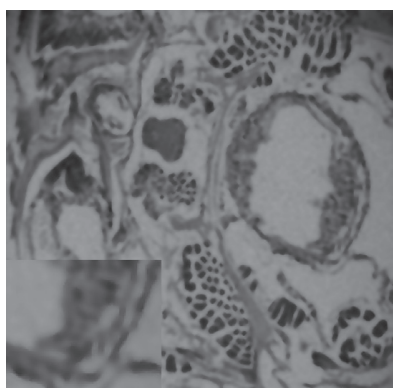

(i)

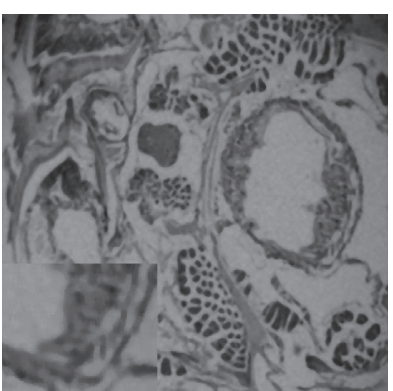

(c)

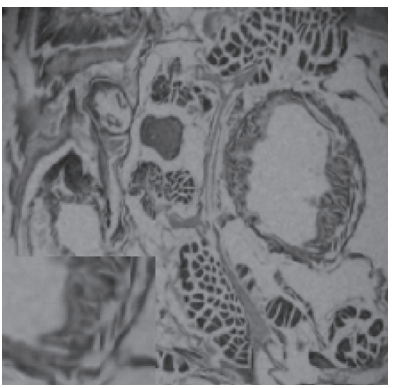

(g)

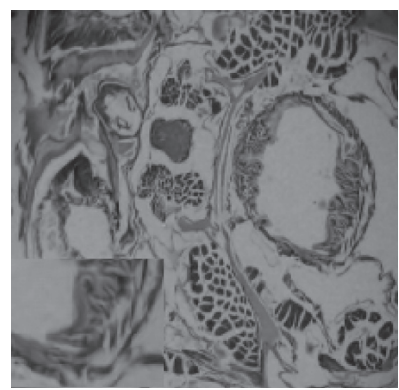

(j)

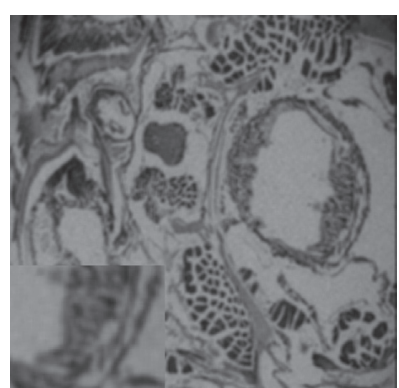

(d)

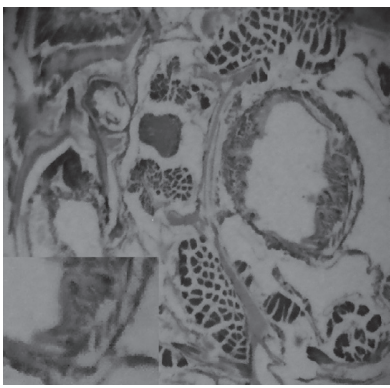

(h)

Figure 10: Restoration results of various algorithms for the locust slice image with Gaussian noise: (a) original image; (b) noise corrupted image; (c) IMED; (d) MEA; (e) WI; (f) WA; (g) INLM; (h) BI; (i) MWI; (j) proposed algorithm.

TABle 4: Comparison with other Gaussian noise removal methods, showing PSNR values for varying amounts of Gaussian noise.

\begin{tabular}{lcccccccc}
\hline$\sigma(\mathrm{dB})$ & IMED & MEA & WI & WA & BI & INLM & MWI & Proposed \\
\hline 0.001 & 29.018 & 27.350 & 30.366 & 28.794 & 27.372 & 30.7461 & 27.557 & $\mathbf{3 1 . 9 2 9}$ \\
0.002 & 28.598 & 27.259 & 29.767 & 28.035 & 27.514 & 29.6031 & 27.309 & $\mathbf{3 1 . 1 9 3}$ \\
0.003 & 28.284 & 27.180 & 29.305 & 27.643 & 27.194 & 27.7093 & 27.129 & $\mathbf{3 0 . 6 1 4}$ \\
0.004 & 27.993 & 27.083 & 28.910 & 27.375 & 26.985 & 26.3833 & 26.965 & $\mathbf{3 0 . 0 5 1}$ \\
0.005 & 27.715 & 26.983 & 28.592 & 27.167 & 25.218 & 25.2624 & 26.800 & $\mathbf{2 9 . 5 3 8}$ \\
\hline
\end{tabular}

TABle 5: Comparison with other Gaussian noise removal methods, showing SSIM values for varying amounts of Gaussian noise.

\begin{tabular}{lcccccccc}
\hline$\sigma(\mathrm{dB})$ & IMED & MEA & WI & WA & BI & INLM & MWI & Proposed \\
\hline 0.001 & 0.814 & 0.775 & 0.839 & 0.806 & 0.867 & 0.8341 & 0.762 & $\mathbf{0 . 8 9 7}$ \\
0.002 & 0.793 & 0.768 & 0.824 & 0.779 & 0.853 & 0.8054 & 0.752 & $\mathbf{0 . 8 7 0}$ \\
0.003 & 0.779 & 0.763 & 0.811 & 0.763 & 0.830 & 0.7614 & 0.745 \\
0.004 & 0.766 & 0.757 & 0.801 & 0.750 & 0.755 & 0.7074 & 0.739 \\
0.005 & 0.753 & 0.751 & 0.791 & 0.741 & 0.731 & 0.6322 & 0.732 & $\mathbf{0 . 8 4 9}$ \\
& & & & &
\end{tabular}




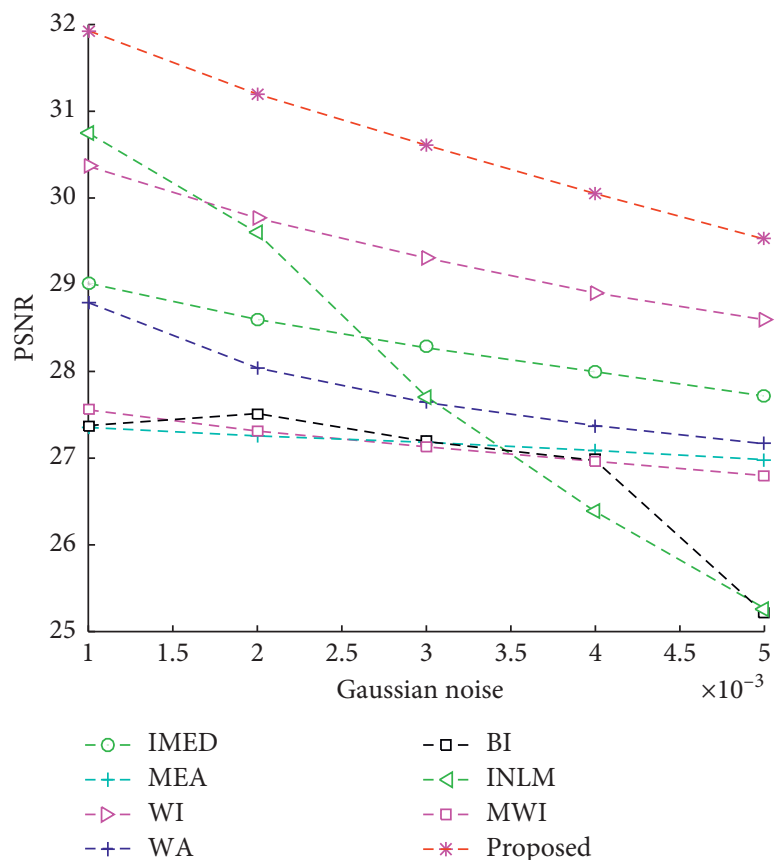

(a)

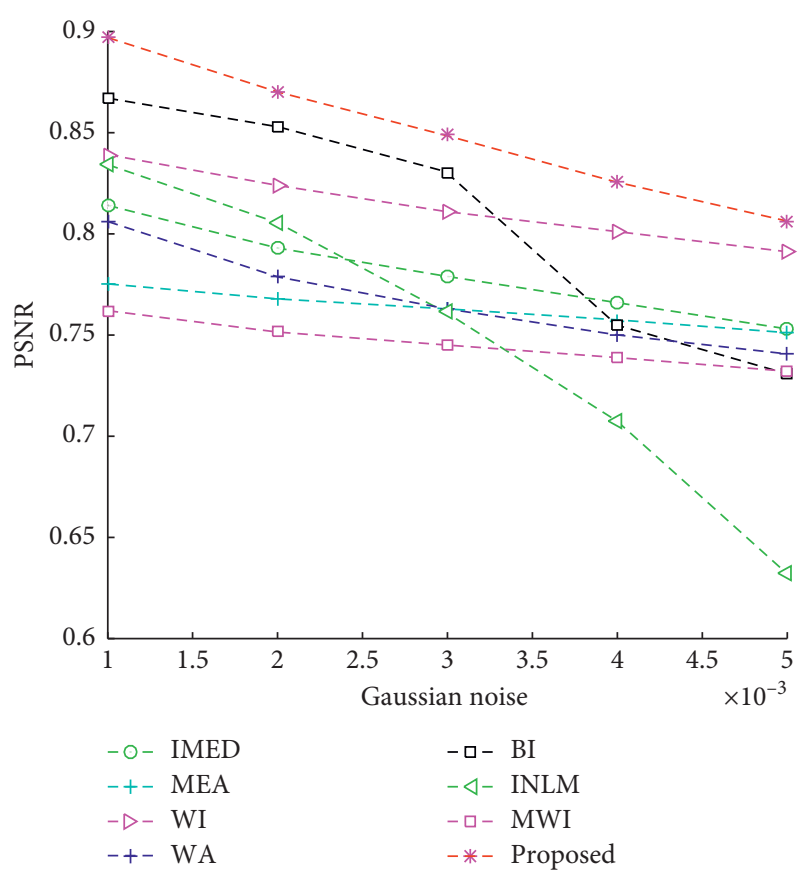

(b)

FIGURE 11: Variation in (a) PSNR and (b) SSIM with varying amounts of Gaussian noise.

proposed algorithm has a stronger capability than other compared denoising algorithms for preserving image structures. Hence, Tables 6 and 7 and Figure 13 indicate that the proposed denoising algorithm is able to more effectively remove mixed noise than other compared denoising algorithms. The ability to remove the mixed noise of the locust slice image from the objective effect is very good. And the structures in the restoration results of the proposed algorithm can be preserved more completely than other algorithms. The proposed algorithm provides important features for identifying targets in locust bioslice images.

4.2. Visual Evaluation of Denoising Performance. Due to the large number of test images, partial noise recovery results are used to evaluate visual effects in each set of experiments. Figure 14 shows the recovery results of various denoising algorithms in the first set of experiments. And the test images are corrupted by salt-and-pepper noise with 0.01 noise density. The original images in these experiments are shown in Figure 1. It can be found that the WI, BI, and INLM fail to restore the noise corrupted image. MEA, WA, and MG suffer from a great quantity of blurred effects for edges and details. MWI suffers from an excessive smoothing effect for restored image. IMED and proposed algorithm can efficiently remove the noise and be free from the blurred effect in the restored images. But the proposed algorithm has no blurred effect in the restored image. So, the proposed algorithm achieves the better visual effect than other denoising algorithms. The proposed algorithm can retain more texture details than the other compared algorithms, especially for the marked area.
Figure 10 shows the recovery results of various denoising algorithms in the second set of experiments. And the test image is corrupted by Gaussian noise with 0.001 noise density. The original image in this experiment is shown in Figure 10(a). Although other algorithms can remove the Gaussian noise from the corrupted images, they still suffer from the blurred effect for the edges and details in the restored images. MEA has a certain effect of removing Gaussian noise, but it makes the image blurred. The INLM and BI can not only filter the Gaussian noise well but also protect the edge information of the image to the greatest extent and achieve better filtering effect. However, the proposed algorithm can retain more texture details than the INLM and BI, especially for the marked area. And, the proposed algorithm has a higher image clarity after restoration.

Figure 12 shows the recovery results of various denoising algorithms in the third set of experiments. And the test image is corrupted by salt-and-pepper noise with a noise intensity of 0.01 and Gaussian noise with standard deviation of 0.002. Original image in this experiment is shown in Figure 12(a). It can be seen from the figure that MEA, WI, WA, and BI are well restored by single noise corruption. These algorithms suffer from blurring effect in the restored image. And there are still some noise points after filtering. The BI can restore image edges and details from the noise corrupted image, and plenty of residual noise still exists in the restored images. The INLM has a certain effect of removing noise and can retain the edge details of the image to the greatest extent. However, the filtered image appears blurred and has some noise points. Although MWI can remove the mixed noise from the corrupted images, they still 


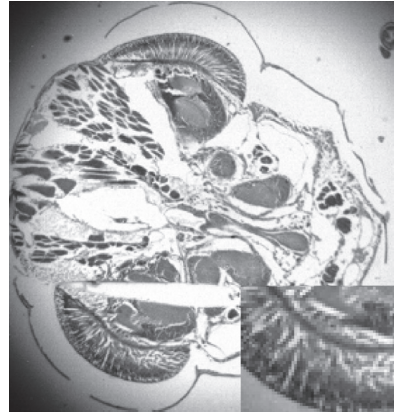

(a)

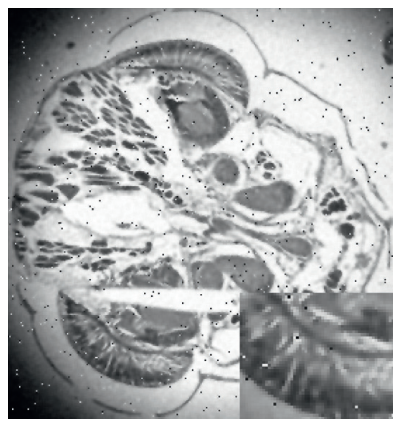

(e)

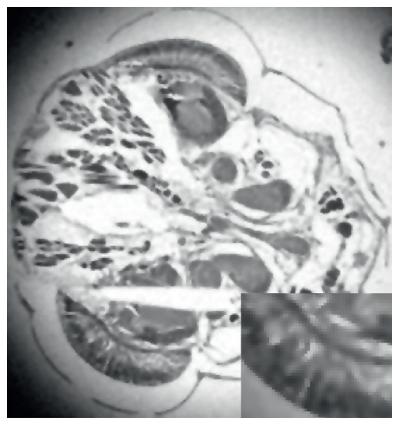

(i)

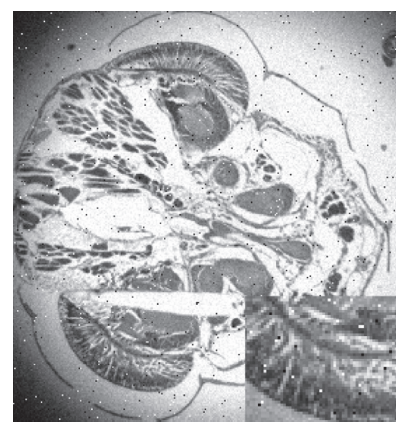

(b)

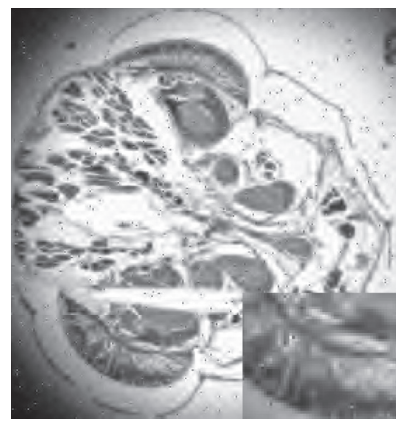

(f)

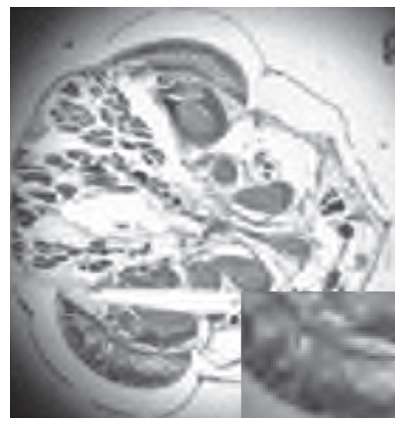

(j)

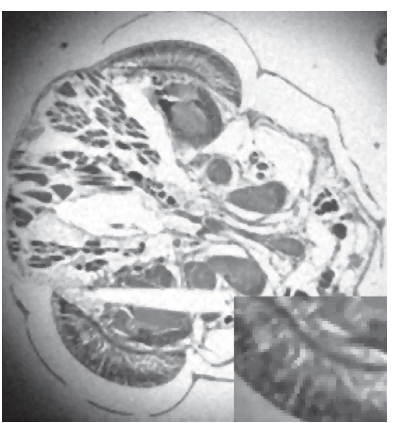

(c)

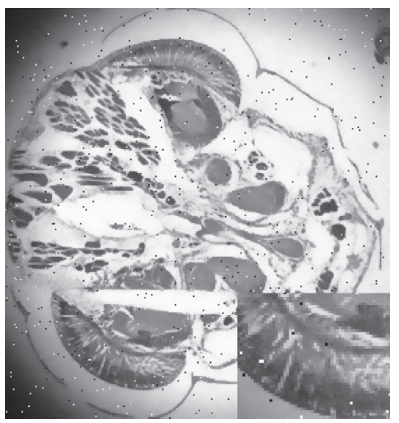

(g)

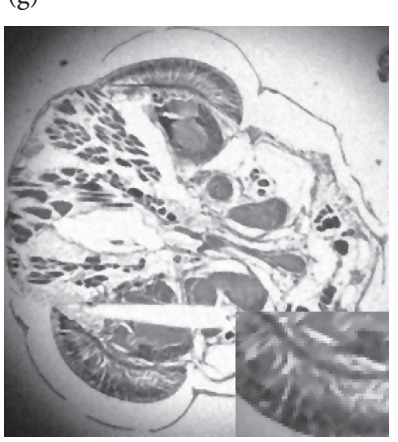

(k)

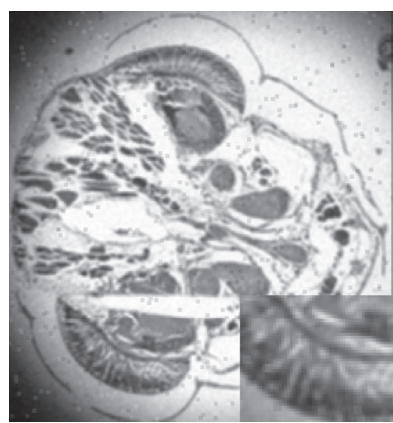

(d)

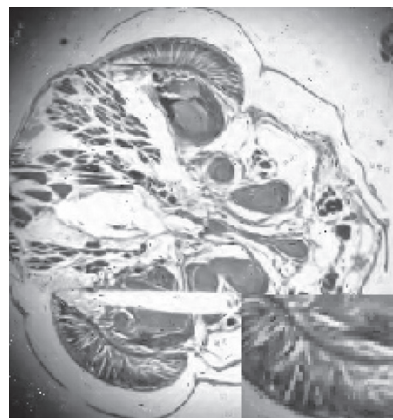

(h)

FIGURE 12: Restoration results of various algorithms for the locust slice image with salt-and-pepper and Gaussian noises: (a) original image; (b) noise corrupted image; (c) IMED; (d) MEA; (e) WI; (f) WA; (g) BI; (h) INLM; (i) MWI; (j) MWA; (k) proposed algorithm.

TABLE 6: Comparison with other mixed noise removal methods, showing PSNR values for varying amounts of salt-and-pepper and Gaussian noises.

\begin{tabular}{lcccccccccc}
\hline $\mathrm{sp}(\mathrm{dB})$ & $\sigma$ & MEA & WI & WA & BI & IMED & INLM & MWI & WAA & Proposed \\
\hline \multirow{3}{*}{0.01} & 0.001 & 24.231 & 24.082 & 23.408 & 23.708 & 26.300 & 24.571 & 25.300 & 22.763 & $\mathbf{2 7 . 2 9 1}$ \\
& 0.002 & 24.083 & 23.823 & 22.923 & 23.382 & 25.842 & 23.2302 & 25.055 & 22.337 & $\mathbf{2 6 . 6 9 0}$ \\
& 0.003 & 23.938 & 23.745 & 22.610 & 23.352 & 25.466 & 22.019 & 24.839 & 22.065 & $\mathbf{2 6 . 0 3 9}$ \\
\hline \multirow{3}{*}{0.02} & 0.001 & 23.781 & 22.237 & 22.725 & 21.319 & 25.862 & 22.2007 & 25.223 & 22.686 & $\mathbf{2 6 . 1 5 7}$ \\
& 0.002 & 23.518 & 21.899 & 22.311 & 20.945 & 25.682 & 20.9751 & 24.968 & 22.248 & $\mathbf{2 5 . 8 2 1}$ \\
& 0.003 & 23.464 & 21.927 & 22.180 & 20.929 & 25.362 & 19.8587 & 24.765 & 22.002 & $\mathbf{2 5 . 3 8 7}$ \\
\hline \multirow{3}{*}{0.03} & 0.001 & 23.238 & 20.868 & 21.966 & 19.561 & 25.537 & 21.1365 & 25.163 & 22.597 & $\mathbf{2 5 . 7 0 3}$ \\
& 0.002 & 23.211 & 21.013 & 21.919 & 19.724 & 25.262 & 19.9516 & 24.947 & 22.215 & $\mathbf{2 5 . 3 1 9}$ \\
& 0.003 & 23.054 & 20.995 & 21.796 & 19.699 & $\mathbf{2 5 . 0 9 4}$ & 18.9311 & 24.728 & 21.952 & 24.731 \\
\hline
\end{tabular}


TABLE 7: Comparison with other mixed noise removal methods, showing SSIM values for varying amounts of salt-and-pepper and Gaussian noises.

\begin{tabular}{lcccccccccc}
\hline sp $(\mathrm{dB})$ & $\sigma$ & MEA & WI & WA & BI & IMED & INLM & MWI & WAA & Proposed \\
\hline \multirow{3}{*}{0.01} & 0.001 & 0.764 & 0.768 & 0.714 & 0.781 & 0.836 & 0.8134 & 0.817 & 0.710 & $\mathbf{0 . 8 5 7}$ \\
& 0.002 & 0.750 & 0.750 & 0.691 & 0.766 & 0.808 & 0.7332 & 0.803 & 0.687 & $\mathbf{0 . 8 1 8}$ \\
& 0.003 & 0.739 & 0.741 & 0.674 & 0.763 & 0.779 & 0.6516 & 0.788 & 0.668 & $\mathbf{0 . 7 8 4}$ \\
\hline \multirow{2}{*}{0.02} & 0.001 & 0.729 & 0.689 & 0.668 & 0.680 & 0.804 & 0.7847 & 0.815 & 0.707 & $\mathbf{0 . 8 2 3}$ \\
& 0.002 & 0.709 & 0.668 & 0.641 & 0.665 & 0.801 & 0.6899 & 0.700 & 0.681 & $\mathbf{0 . 8 1 0}$ \\
& 0.003 & 0.704 & 0.663 & 0.640 & 0.660 & 0.776 & 0.5957 & 0.786 & 0.665 & $\mathbf{0 . 7 9 3}$ \\
\hline \multirow{3}{*}{0.03} & 0.001 & 0.691 & 0.621 & 0.616 & 0.592 & 0.783 & 0.7675 & 0.815 & 0.704 & $\mathbf{0 . 7 9 9}$ \\
& 0.002 & 0.687 & 0.622 & 0.614 & 0.602 & 0.758 & 0.6621 & 0.799 & 0.680 & $\mathbf{0 . 7 6 4}$ \\
& 0.003 & 0.676 & 0.615 & 0.608 & 0.595 & 0.724 & 0.566 & 0.786 & 0.663 & $\mathbf{0 . 7 4 0}$ \\
\hline
\end{tabular}

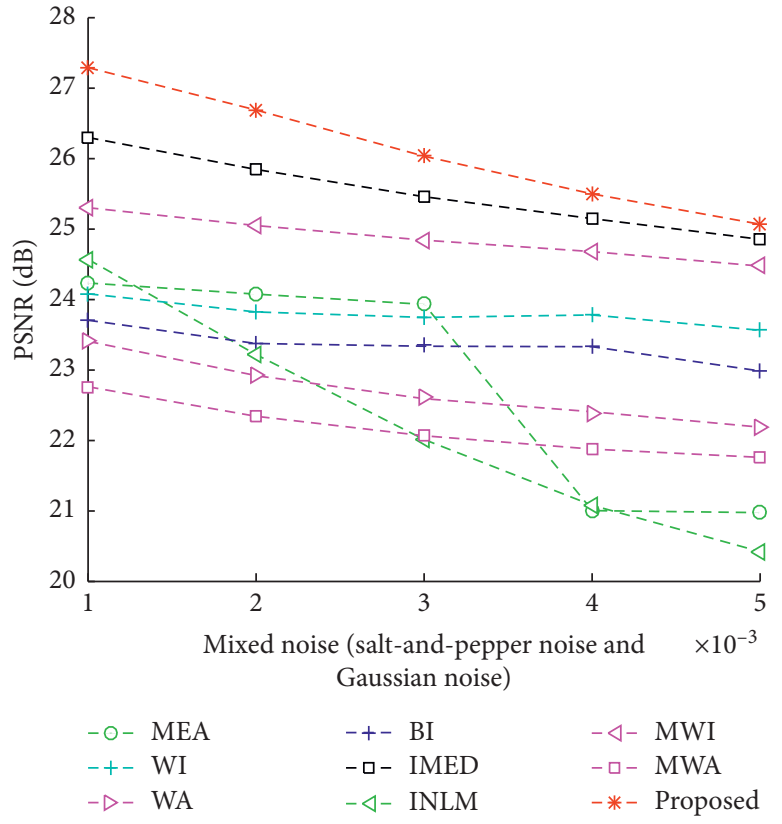

(a)

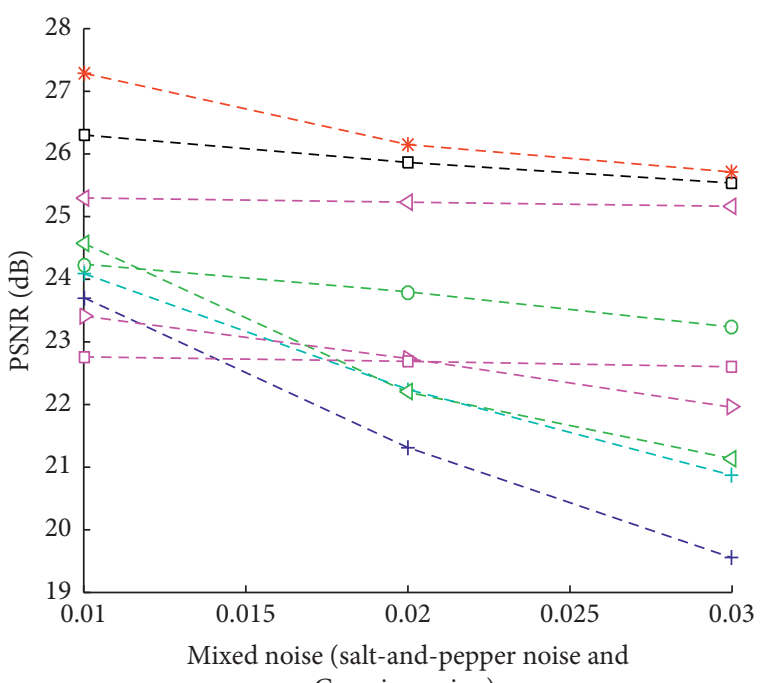

Gaussian noise )

$\begin{array}{lll}-\circ-\text { MEA } & -+- \text { BI } & -\triangleleft-\text { MWI } \\ -+- \text { WI } & -\square-\text { IMED } & -\square-\text { MWA } \\ -\triangleright-\text { WA } & -\triangleleft-\text { INLM } & -*-\text { Proposed }\end{array}$

(b)

FIGURE 13: Variation in PSNR with varying amounts of salt-and-pepper noise and Gaussian noises: (a) $\mathrm{sp}=0.01, \sigma$ varying from 0.001 to 0.005 ; (b) $\sigma=0.001$, sp varying from 0.01 to 0.03 .

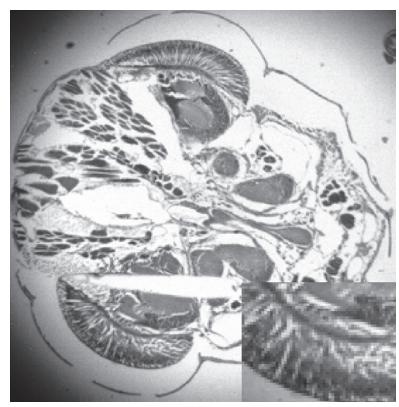

(a)

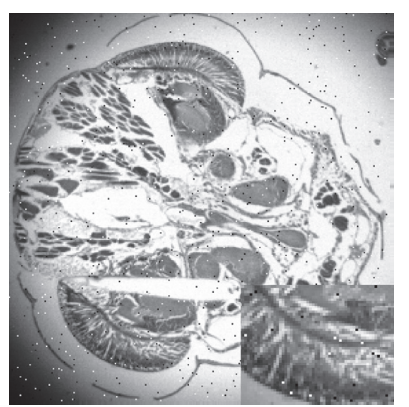

(b)

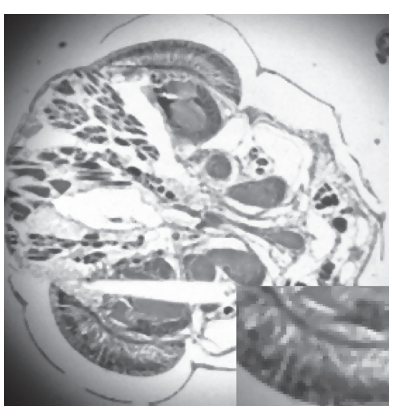

(c)

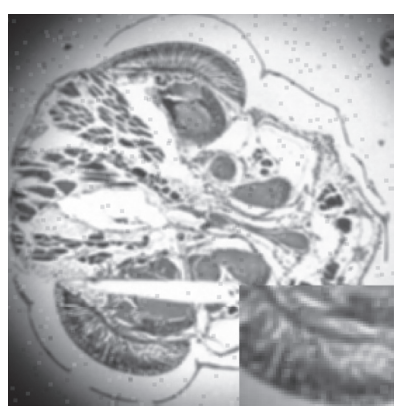

(d)

FIgURE 14: Continued. 


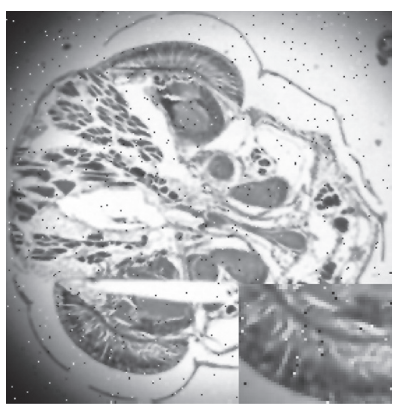

(e)

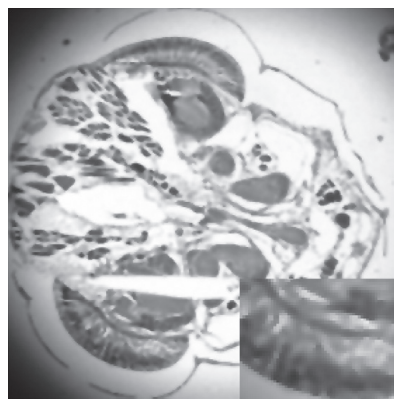

(i)

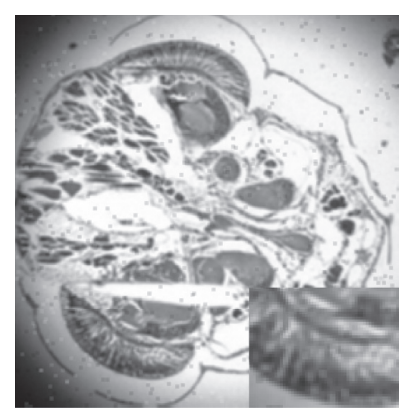

(f)

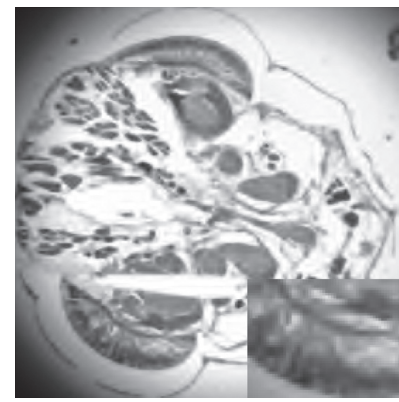

(j)

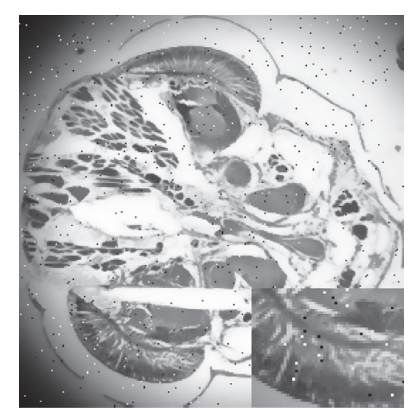

(g)

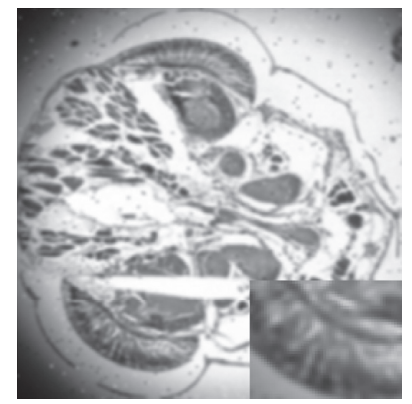

(k)

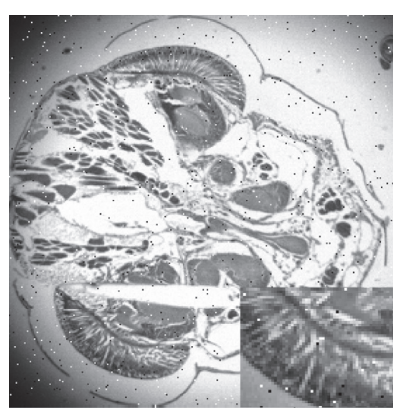

(h)

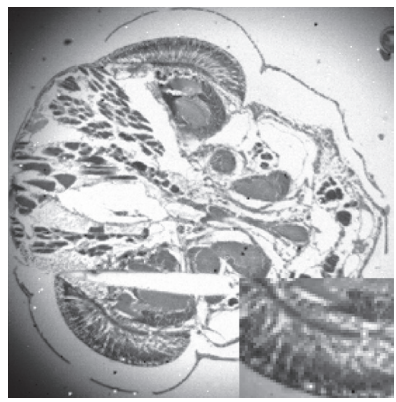

(1)

FIGURE 14: Restoration results of various algorithms for the locust slice image with salt-and-pepper noise: (a) original image; (b) noise corrupted image; (c) IMED; (d) MEA; (e) WI; (f) WA; (g) BI; (h) INLM; (i) MWI; (j) MWA; (k) MG; (l) proposed algorithm.

suffer from the blurred effect for the edges and details in the restored images. The proposed algorithm can efficiently remove the noise and be free from the blurred effect in the restored images. Besides, the proposed algorithm achieves the better visual effect than other algorithms because they can preserve more edges and contain less noise in the restoration images. Therefore, the above restoration results clearly indicate that the proposed algorithm obtains the best visual effect in terms of noise suppression and detail preservation, especially for the marked area.

\section{Conclusions}

Shannon-cosine wavelet precise integration method is a new image denoising method based on Shannon-cosine wavelet and the nonlinear PDE variational model, which was used to construct adaptive wavelet interpolation operator due to multiscale characteristics of wavelet transform. The adaptive interpolation operator in the Shannon-cosine wavelet precision integration method can reduce the amount of the collocation points and improve the calculation efficiency. The efficiency is about $86 \%$ higher than the original efficiency. Multiscale wavelet transform coefficients are used to identify and remove salt-and-pepper noise in slice images. The proposed algorithm shows superior denoising performance to representative denoising algorithms.

The method makes full use of the multiscale characteristics and the high precise performance of precise integration method. Based on the edge retention smoothing model, SWPIM is used to remove Gaussian noise in biomedical images. The precise integration method is used to calculate the nonlinear ordinary differential equations, which improves the accuracy of the calculation. Compared to the other methods of image denoising, object boundary of locust slice images obtained by SWPIM denoising method is clear. With the increase in noise, the proposed algorithm can achieve the larger values of PSNR and SSIM than other algorithms. Experimental results show that the proposed calculation method of adaptive selection parameter $k$ can accurately identify Gaussian noise and image contours in the image. SWPIM method is very suitable for measurement of image denoising, such as locust slice images.

\section{Data Availability}

The locust slice data used to support the findings of this study have been deposited in the Locusts-slice-data repository (https://github.com/feifei123feifei/Locusts-slicedata. git). All data generated or analyzed during this study are included in these files.

\section{Conflicts of Interest}

The authors declare that they have no conflicts of interest.

\section{Acknowledgments}

The authors would like to thank their colleagues for all their help and support during this work. This work was supported by the Beijing Natural Science Foundation (Grant no. 4172034) and National Natural Science Foundation of China (Grant no. 61871380). 


\section{References}

[1] L. Li, N. Zhang, S. Mei, and X. Li, "Image denoising of locust sections based on adaptive wavelet and partial differential equation method," Transactions of the Chinese Society of Agricultural Engineering, vol. 31, no. 20, pp. 172-177, 2015.

[2] L. Li, S. Guo, S. Mei, and N. Zhang, "Image restoration of locust slices based on nearest unit matching," Transactions of the Chinese Society of Agricultural Machinery, vol. 46, no. 8, pp. 15-19, 2015.

[3] S. Mei, "Denoising for locust slice image with texture preserving based on coupling technology of variational method and shearlet transform," Transactions of the Chinese Society of Agricultural Engineering, vol. 32, no. 17, pp. 152-159, 2016.

[4] W. Tian, T. Ma, Y. Zheng et al., "Weighted curvature-preserving PDE image filtering method," Computers \& Mathematics with Applications, vol. 70, no. 6, pp. 1336-1344, 2015.

[5] G. Liu and W. Guo, "Application of improved arithmetic of median filtering denoising," Computer Engineering and Applications, vol. 46, no. 10, pp. 187-189, 2010.

[6] S. Yi and J. He, "Image denoising method based on bemd and adaptive wiener filter," Computer Engineering and Applications, vol. 49, no. 10, pp. 156-158, 2013.

[7] X. Liu, Y. Tian, H. Hui, and Y. Zhong, "Improved non-local means algorithm for image denoising," Computer Engineering, vol. 38, no. 4, pp. 199-201, 2012.

[8] X. Qian and L. Guo, "Adaptive Gaussian filter based on object scale," Computer Engineering and Applications, vol. 46, no. 12, pp. 14-20, 2010.

[9] R. Xing and S. Mei, "Engineering drawings denoising based on orthogonal wavelet packets transormation," Journal of China Agricultural University, vol. 8, no. 6, pp. 62-65, 2003.

[10] B. Xiong and Z. Yin, "A universal denoising framework with a new impulse detector and nonlocal means," IEEE Transactions on Image Processing, vol. 21, no. 4, pp. 1663-1675, 2011.

[11] J. Zhang, L. Pei, X. Li, and H. Wu, "Hybrid denoising algorithm combining median filtering with sparse representation," Computer Engineering, vol. 42, no. 9, pp. 240-245, 2016.

[12] B. Smolka and D. Kusnik, "Robust local similarity filter for the reduction of mixed Gaussian and impulsive noise in color digital images," Signal, Image and Video Processing, vol. 9, no. 1, pp. 49-56, 2015.

[13] H. Chen, Y. Zhang, M. K. Kalra et al., "Low-dose CT with a residual encoder-decoder convolutional neural network (RED-CNN)," IEEE Transactions on Medical Imaging, vol. 36, 2017.

[14] L. Li, N. Zhang, and S. Mei, "Anisotropic dynamic diffusion model for texture preserving de-noising of tomato images," Transactions of the Chinese Society for Agricultural Machinery, vol. 47, no. 11, pp. 18-24, 2016.

[15] C. Cattani, "Shannon wavelets theory," Mathematical Problems in Engineering, vol. 2008, Article ID 16480, 24 pages, 2008.

[16] C. Cattani, A. Ciancio, and B. Lods, "On a mathematical model of immune competition," Applied Mathematics Letters, vol. 19, no. 7, pp. 678-683, 2006.

[17] A. Aldroubi, M. Unser, and A. Aldroubi, "Sampling procedures in function spaces and asymptotic equivalence with shannon's sampling theory," Numerical Functional Analysis and Optimization, vol. 15, no. 1-2, pp. 1-21, 1994.

[18] D. K. Hoffman, G. W. Wei, D. S. Zhang, and D. J. Kouri, "Shannon-gabor wavelet distributed approximating functional," Chemical Physics Letters, vol. 287, no. 1-2, pp. 119-124, 1998.
[19] G. W. Wei, "Quasi wavelets and quasi interpolating wavelets," Chemical Physics Letters, vol. 296, no. 3-4, pp. 215-222, 1998.

[20] S.-L. Mei, H.-L. Lv, and Q. Ma, "Construction of interval wavelet based on restricted variational principle and its application for solving differential equations," Mathematical Problems in Engineering, vol. 2008, Article ID 629253, 14 pages, 2008.

[21] H. Wang and S.-L. Mei, "Shannon wavelet precision integration method for pathologic onion image segmentation based on homotopy perturbation technology," Mathematical Problems in Engineering, vol. 2014, no. 3, 10 pages, Article ID 601841, 2014.

[22] S. Mei and W. Gao, "Shannon-cosine wavelet spectral method for solving fractional Fokker-Planck equations," International Journal of Wavelets, Multiresolution and Information Processing, vol. 16, no. 3, Article ID 1850021, 2018.

[23] Y. Wei, Y. Zhang, S. Mei, and S. Wei, "Image dehazing method based on dark channel prior and interval interpolation wavelet transform," Transactions of the Chinese Society of Agricultural Engineering, vol. 33, no. 1, pp. 281-287, 2017.

[24] S. Guo, S. Mei, and L. Li, "Texture extension method for farmland remote sensing image based on shannon-cosine wavelet," Transactions of the Chinese Society for Agricultural Machinery, vol. 48, no. S1, pp. 142-146, 2017.

[25] Y. Zhang, Y. Wei, S. Mei, and M. Zhu, "Application of multiscale interval interpolation wavelet in beef image of marbling segmentation," Transactions of the Chinese Society of Agricultural Engineering, vol. 32, no. 21, pp. 296-304, 2016.

[26] J. L. Wu, "A wavelet operational method for solving fractional partial differential equations numerically," Applied Mathematics and Computation, vol. 214, no. 1, pp. 31-40, 2009.

[27] S. Mei, "Adaptive wavelet precise integration method on nonlinear Black-Scholes equations," Journal of Quantitative Economics, vol. 29, no. 04, pp. 8-14, 2012.

[28] S. Mei, X. Li, H. Zhao, Li Li, and S. Guo, "Method of denoising and removing artifacts for farm remote sensing image based on shearlet and total variation," Transactions of the Chinese Society of Agricultural Engineering, vol. 33, no. 1, pp. 274-280, 2017.

[29] H. Wang, M. Zhu, Li Li, L. Wang, H. Zhao, and S. Mei, "Regional weed identification method from wheat field based on unmanned aerial vehicle image and Shearlets," Transactions of the Chinese Society of Agricultural Engineering, vol. 33, no. 1, pp. 99-106, 2017.

[30] S.-L. Mei and D. Zhu, "HPM-based dynamic sparse grid approach for perona-malik equation," The Scientific World Journal, vol. 2014, Article ID 417486, 15 pages, 2014.

[31] S. Mei, "HPM-based dynamic wavelet transform and its application in image denoising," Mathematical Problems in Engineering, vol. 2013, Article ID 309418, 10 pages, 2013.

[32] X. Li, S. Mei, and L. Li, "Image menoising of locust slices based on Meyer window function shearlet transform," Transactions of the Chinese Society of Agricultural Engineering, vol. 47, no. z1, pp. 449-456, 2016. 